\title{
Review of Emotion Recognition in Stroke Patients
}

\author{
Rajamanickam Yuvaraj ${ }^{a}$ Murugappan Murugappan ${ }^{a}$ \\ Mohamed Ibrahim Norlinah ${ }^{b}$ Kenneth Sundaraj ${ }^{a} \quad$ Mohamad Khairiyah $^{b}$ \\ a School of Mechatronic Engineering, University Malaysia Perlis (UniMAP), Arau, and \\ ${ }^{b}$ Neurology Unit, Department of Medicine, UKM Medical Center, Kuala Lumpur, Malaysia
}

\section{Key Words}

Emotion recognition - Emotion processing · Brain damage patients · Communication channels · Neuropsychology

\begin{abstract}
Objective: Patients suffering from stroke have a diminished ability to recognize emotions. This paper presents a review of neuropsychological studies that investigated the basic emotion processing deficits involved in individuals with interhemispheric brain (right, left) damage and normal controls, including processing mode (perception) and communication channels (facial, prosodic-intonational, lexical-verbal). Methods: An electronic search was conducted using specific keywords for studies investigating emotion recognition in brain damage patients. The PubMed database was searched until March 2012 as well as citations and reference lists. 92 potential articles were identified. Results: The findings showed that deficits in emotion perception were more frequently observed in individuals with right brain damage than those with left brain damage when processing facial, prosodic and lexical emotional stimuli. Conclusion: These findings suggest that the right hemisphere has a unique contribution in emotional processing and provide support for the right hemisphere emotion hypothesis. Significance: This robust deficit in emotion recognition has clinical significance. The extent of emotion recognition deficit in brain damage patients appears to be correlated with a variety of interpersonal difficulties such as complaints of frustration in social relations, feelings of social discomfort, desire to connect with others, feelings of social disconnection and use of controlling behaviors.


Dementia

Cognitive Disorders

\begin{tabular}{l|l}
\hline Dement Geriatr Cogn Disord 2013;36:179-196 \\
\hline DOI: $\underline{10.1159 / 000353440}$ & $\begin{array}{l}\text { @ 2013 S. Karger AG, Basel } \\
\text { www.karger.com/dem }\end{array}$ \\
\hline
\end{tabular}

Yuvaraj et al.: Review of Emotion Recognition in Stroke Patients

\section{Introduction}

Stroke is an increasingly common condition which significantly affects the life of a patient on many levels. It is the third leading cause of death in developed and developing countries, and it was estimated to cause 5.5 million deaths worldwide, which is equivalent to $9.6 \%$ of all deaths [1]. Stroke causes impaired motor control, language and memory impairments, and produces a disruption in a patient's ability to perceive emotions [2]. Over the last two decades, the study of emotional processing following stroke has received major attention in the emotion literature. Here, the term 'emotion perception' involves the identification of emotional information in the environment across multiple channels of communication (facial, prosodic and lexical). Decoding emotion signals (e.g., the face) is considered to be an important part of social interactions. Research has identified numerous neuroanatomical structures and neurophysiological systems that modulate emotion at the cortical, subcortical and limbic levels of the nervous system [3]. Many successful paradigms have been used to study emotional processing including behavioral techniques (dichotic listening and tachistoscopic viewing), or expressions (facial asymmetry, vocal, verbal and gestural/postural), brain lesion methods (studies of individuals with known brain damage), neurosurgical procedures and functional neuroimaging techniques [3]. The purpose of this review was to examine the literature on hemispheric specialization of emotion processing. Findings from experimental studies with normal controls and individuals with rightor left-sided brain damage with respect to three communication channels (face, voice and lexical perception) will be summarized. Wherever available, valence-specific findings will be reported. This growing body of research has potential implications for the assessment of emotional disorders in patients that are associated with right hemisphere stroke, including depression, anxiety, anger and/or mania [4], and with left hemisphere stroke, including aphasia [5].

\section{Theories of Hemispheric Specialization of Emotion}

Hemispheric specialization refers to the differentiation of higher functions between the two hemispheres; its study is based on findings in which certain functions are predominantly affected by damage to one hemisphere [6]. There are two major hypotheses concerning hemispheric specialization for emotion.

The first hypothesis is known as the 'right hemisphere hypothesis', which proposes that the right hemisphere plays a greater role in emotion processing regardless of valence $[7,8]$. Emotional processing at the behavioral level involves strategies (nonverbal, integrative, synthetic, holistic and gestalt) and functions (pattern perception, visuospatial organization and visual imaging) for which the neuroanatomical design and the neuropsychological organization of the right hemisphere are better suited [6]. The right cerebral hemisphere has been described as having greater ability to perform intermodal integration [9], greater interlobular organization [10], stimulus-evoked physiological activity [11] and functional interconnectivity among regions compared to the left hemisphere [12]. Heilman [13] suggested that the right hemisphere is more in touch with subcortical systems that are important for emotion processing. Tucker and Williamson [14] argue that the right hemisphere's specialized processing features lend themselves to nonverbal (e.g., emotional) processing, whereas the left hemisphere's features lend themselves to verbal (e.g., cognitive) processing. Support also comes from studies in which right hemisphere pathology was more involved in emotion processing. This has been associated with abnormal patterns of autonomic nervous system responses, as measured by heart rate [15] and skin conductance [16].

The second hypothesis is known as the 'valence hypothesis', which proposes that the right hemisphere is specialized for negative or unpleasant emotions, and the left hemisphere is 
specialized for positive or pleasant emotions [14, 17, 18]. Borod et al. [19] suggested that the quality of decision making is different in the case of negative compared to positive emotions. Since negative emotions are linked with survival, a system is required that is sensitive to multimodal inputs and that quickly scans the environment and evaluates the situation. This type of behavior seems to be more linked to right than to left hemisphere function. Positive emotions, on the other hand, may be more dominated by the left hemisphere. Borod et al. [20] suggested that positive emotions often are more linguistic and communicative than emotional and reactive, thus possibly reflecting left hemisphere involvement in their function. It has also been proposed that social emotions (e.g., affection, pride, embarrassment) are modulated by the left hemisphere whereas primary emotions (e.g., anger, fear, sadness) are modulated by the right hemisphere [21]. Two studies involving patients with brain damage have shown an involvement for patients with right hemisphere brain damage (RBD) in social emotions [22] and impairment for patients with left hemisphere brain damage (LBD) in this domain [23].

\section{Methods}

\section{Criteria for Study Inclusion}

Types of Studies. Only studies comparing emotion recognition performance in a group of participants with brain damage to that in a normal control group were used for the purposes of this review. Emotion recognition was defined as any task in which stimuli conveying emotional information were presented, and for which participants were asked to state or choose which emotion they thought was represented by the stimulus. The stimulus could be facial, prosodic or lexical and of any form (e.g., static faces, videos, recorded voices). Any target emotions were considered. Studies looking exclusively at emotion production rather than the recognition of emotions, or studies which focused on neuroanatomy rather than behavioral performance (e.g., discrimination task measures) or semantic knowledge (e.g., situations that might be expected to induce emotions) were excluded. Reviews, editorials, letters or other articles that did not contain original data were excluded as well. The methodology scheme for the selection of articles is shown in detail in figure 1.

Types of Participants. The patient group consisted of participants whose brain damage resulted from stroke, confirmed by neurologists and/or radiologists based on neuroimaging results using either computed tomography or magnetic resonance imaging. Studies of both right and left brain damage participants were included. The control group consisted of neurologically healthy participants. Only data from adult participants over the age of 18 were considered.

\section{Electronic Search}

We conducted a systematic electronic search in the PubMed database, which included Taylor \& Francis, PsycINFO, PsycNET, Science Direct, Wiley's Online Library and SpringerLink. We used the following keywords: 'emotional impairment', 'recognition', 'behavior', 'perception', 'facial expression', 'prosody', 'lexical' and 'emotion comprehension', and limited these searches to stroke or brain damage. This literature search was carried out from December 2011 to July 2012 at the University Malaysia Perlis, Malaysia. The search articles included all relevant papers until March 2012. Only peer-reviewed published articles were accepted for inclusion. Attempts were also made to contact corresponding authors of articles included in the review to either ask for access to more demographic or experimental data or to make queries about particular studies. For each study included in the review, a manual search of references and a citation search were also conducted to identify additional studies.

\section{Study Selection}

Based on these criteria, initial searches identified 966 articles. The title and abstract of each citation were examined independently in the PubMed database for the pre-specified inclusion and exclusion criteria listed above. 127 articles were included based on title and abstract alone. The full text of these papers was obtained through our university library to assess whether they fully met inclusion criteria. Four additional citations were identified from the reference list and citation search. Data were extracted to a data collection form. This covered clinical characteristics, details of emotion recognition tests and results. 


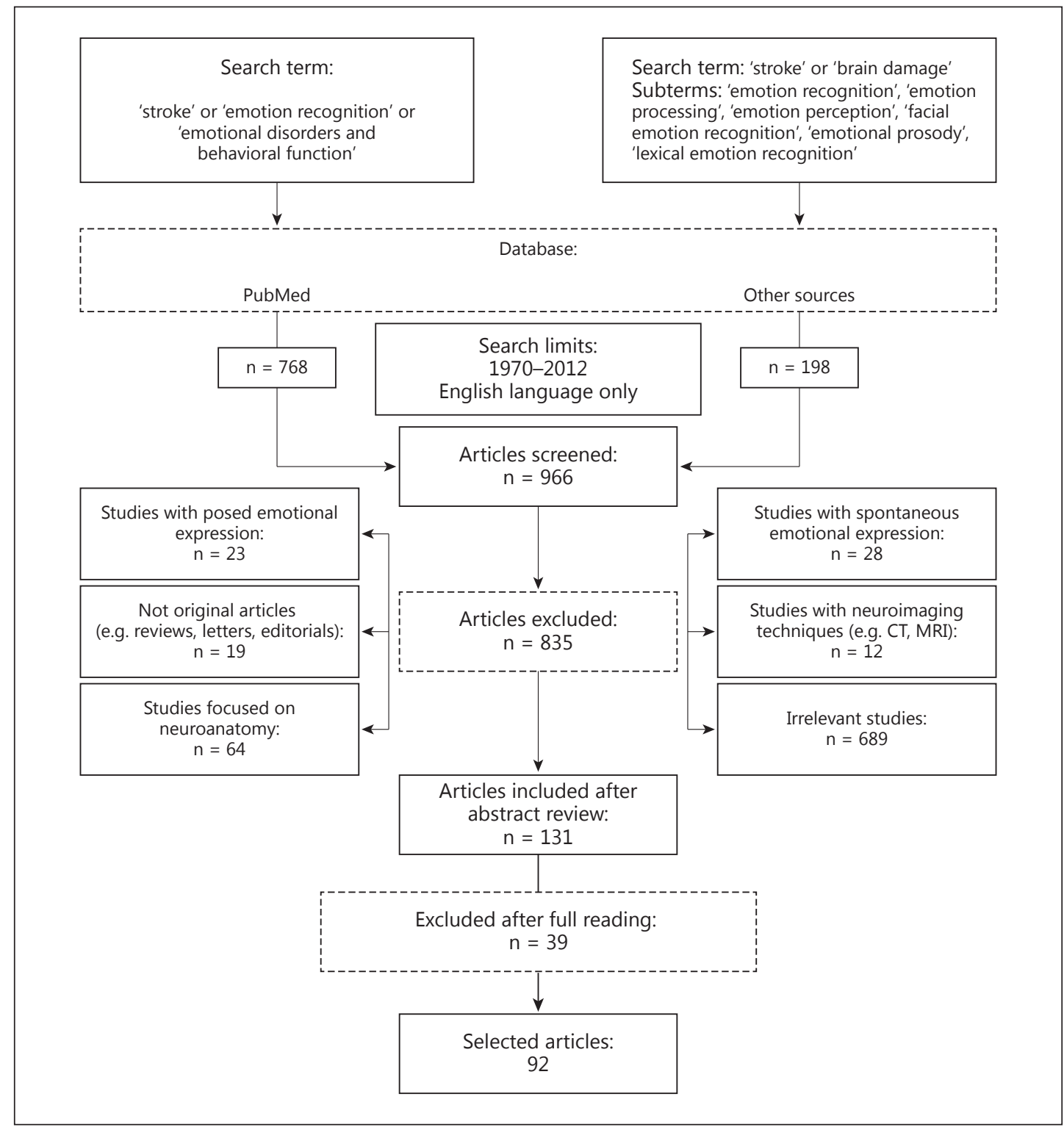

Fig. 1. Methodology scheme for the selection of articles. CT = Computed tomography; MRI = magnetic resonance imaging.

\section{Results}

\section{Search Results}

After the full text of each article had been examined, 92 articles were found to meet the inclusion criteria. The characteristics of the brain damage patients included in these studies are illustrated in tables 1-3.

\section{Assessment of Methodological Factors}

The study methodology was assessed in four distinct categories: participant characteristics, emotional stimuli employed, stimuli presentation and response options, and control tasks. 


\section{Participant Characteristics}

The exclusion criteria reported were generally consistent among the studies. Participants with disorders known to impact upon cognitive status (e.g., history of neurological disorders or other major medical illness) or emotion processing (e.g., psychiatric disorders) were excluded. Participants who had vision disturbance or hearing difficulties were also excluded. In the studies that were reviewed, mean illness duration ranged from 26.5 days [39] to 23 months [43]. The majority of studies had selected patients receiving some form of medication for the treatment of stroke [31, 50,53]. Even among the studies that enrolled patients who were generally receiving medication, there may be substantial 'on-off' variation depending on the time of day and when participants last took their medication. Moreover, some participants may have received optimal medication, whereas others may not have done so. Only one study reported on stroke severity measures [39]. All studies recruited normal controls as a comparison group. Neuropsychological testing was used to assess performance on language, memory and executive functioning. In all studies, the MiniMental State Examination (MMSE) score was a key part of neuropsychological evaluation [24]. One study used a cut-off score of 21 and another a cut-off score of 23 on the MMSE [25, 26].

\section{Emotional Stimuli}

The majority of studies used very similar sets of stimuli. In order to study facial emotion recognition, participants were asked to discriminate, identify or match a range of facial emotional expressions to either photographs, cartoons or line drawing stimuli, depending on the study [25, 30-37]. Studies varied in the use of either black and white [25, 31, 32, 38] or color facial photographs [39]. Most research was done using static stimuli, predominantly from the Pictures of Facial Affect battery [40]. Only a small number of studies employed dynamic images (video recordings) [41, 42]. However, there were a number of variations of this task: some studies used simple facial images [32,54] and some morphed facial images [31], others used neutral faces whereas some did not; additionally, the overall number of stimuli used differed. Some researchers opted to use other validated facial stimuli [34] or worked with their own personally designed stimuli [41,43].

Studies of prosodic emotion recognition often ask participants to discriminate between two emotionally toned sentences that convey a particular emotion and to identify the affect being expressed. These study stimuli were mostly drawn from the New York Emotion Battery (NYEB) database [27, 43, 44], and most studies varied in the use of emotional prosody sentences. Past studies have asked participants to identify or discriminate the recorded emotional prosody category of semantically neutral sentences [26, 28, 29, 45-47], low-pass filtered sentences [48-50], sentence pairs [7, 33, 51, 52], congruent or incongruent sentences [53], pseudo-sentences [30, 41, 54] or hummed utterances [55].

Lexical emotion recognition tasks require individuals to identify the emotion underlying spoken sentences $[22,56,57]$. This pool of emotional items was derived from numerous sources, including dictionaries, word lists and monographs. Few studies attempted to explore all the stimuli in parallel emotional processing [41, 43, 44, 54]. For example, Schmitt et al. [41] investigated the performance under conditions of multimodal channels in terms of facial expression, emotional prosody and the emotional meaning of underlying spoken sentence. After presentation of each item, the participant was required to judge the corresponding emotion, being offered a choice of emotions. With regard to emotion examined, all communication channels were presented with a range of basic emotions, such as happiness, sadness, anger, fear, disgust, surprise, neutral emotion or a combination of these. 
Table 1. Summary of emotion processing studies from the facial channel

\begin{tabular}{|c|c|c|c|c|c|c|c|}
\hline Reference & $\mathrm{n}$ & $\begin{array}{l}\text { Clinical } \\
\text { measures }\end{array}$ & $\begin{array}{l}\text { Control } \\
\text { task }\end{array}$ & Emotional stimuli & Emotions & Dependent variables & Results \\
\hline $\begin{array}{l}\text { Blonder et al. } \\
\text { [33] }\end{array}$ & $\begin{array}{l}12 \mathrm{RBD} \\
9 \mathrm{NC}\end{array}$ & GDS; PANAS & no & static female faces & H, Sa, A, F, N & $\begin{array}{l}\text { discrimination; } \\
\text { matching }\end{array}$ & $\mathrm{RBD}<\mathrm{NC}$ \\
\hline $\begin{array}{l}\text { Harciarek } \\
\text { and Heilman } \\
\text { [32] }\end{array}$ & $\begin{array}{l}11 \mathrm{ASRH} \\
16 \mathrm{PSRH} \\
31 \mathrm{NC}\end{array}$ & MMSE & no & $\begin{array}{l}\text { static photographs } \\
\text { (Pictures of Facial Affect) }\end{array}$ & H, Sa, F, A, Su, D & identification & $\mathrm{ASRH}<\mathrm{PSRH}<\mathrm{NC}$ \\
\hline $\begin{array}{l}\text { Cheung et al. } \\
\text { [31] }\end{array}$ & $\begin{array}{c}19 \mathrm{NC} \\
19 \mathrm{RSS} \\
19 \mathrm{LSS} \\
6 \mathrm{~T} \\
6 \mathrm{BG}\end{array}$ & NA & no & $\begin{array}{l}\text { static photographs } \\
\text { (Pictures of Facial Affect) }\end{array}$ & H, Sa, A, Su, D, F & identification & $\begin{array}{l}\text { in the group, } \\
\text { (i) RSS = LSS = NC, } \\
\text { (ii) } \mathrm{T}<\mathrm{NC}(\mathrm{Sa})\end{array}$ \\
\hline $\begin{array}{l}\text { Harciarek } \\
\text { et al. [54] }\end{array}$ & $\begin{array}{l}30 \mathrm{RBD} \\
31 \mathrm{NC}\end{array}$ & MMSE & no & $\begin{array}{l}\text { static photographs } \\
\text { (Pictures of Facial Affect) }\end{array}$ & $\mathrm{H}, \mathrm{Sa}, \mathrm{A}$ & recognition & $\mathrm{RBD}<\mathrm{NC}$ \\
\hline Braun et al. [39] & $\begin{array}{l}13 \mathrm{RBD} \\
11 \mathrm{LBD} \\
29 \mathrm{NC}\end{array}$ & $\begin{array}{l}\text { SSS; EBI; } \\
\text { ZVT-G; BDI }\end{array}$ & no & static color photographs & $\mathrm{H}, \mathrm{Sa}, \mathrm{F}, \mathrm{A}, \mathrm{Su}, \mathrm{D}$ & $\begin{array}{l}\text { identification; } \\
\text { labeling }\end{array}$ & $\begin{array}{l}\mathrm{RBD}<\mathrm{LBD}<\mathrm{NC} \\
(\mathrm{F}, \mathrm{A}, \mathrm{Sa}, \mathrm{D})\end{array}$ \\
\hline $\begin{array}{l}\text { Yip et al. } \\
\text { [34] }\end{array}$ & $\begin{array}{l}14 \mathrm{SS} \\
14 \mathrm{NC}\end{array}$ & $\begin{array}{l}\text { FDST; BT; } \\
\text { BDST; ST; } \\
\text { VFT; HVOT }\end{array}$ & no & $\begin{array}{l}\text { static photographs } \\
\text { (JACFEE) }\end{array}$ & H, Sa, A, Su, D, F & $\begin{array}{l}\text { identification; } \\
\text { discrimination }\end{array}$ & $\mathrm{SS}<\mathrm{NC}(\mathrm{F}, \mathrm{Sa}, \mathrm{D})$ \\
\hline $\begin{array}{l}\text { Charbonneau } \\
\text { et al. [25] }\end{array}$ & $\begin{array}{l}15 \mathrm{RBD} \\
17 \mathrm{LBD} \\
16 \mathrm{NC}\end{array}$ & MMSE & no & $\begin{array}{l}\text { static photographs } \\
\text { (Pictures of Facial Affect) }\end{array}$ & $\mathrm{H}, \mathrm{Sa}, \mathrm{A}, \mathrm{Su}, \mathrm{D}, \mathrm{F}$ & $\begin{array}{l}\text { discrimination; } \\
\text { identification }\end{array}$ & $\begin{array}{l}\text { facial: } \mathrm{RBD}<\mathrm{LBD}<\mathrm{NC} \\
(\mathrm{H}, \mathrm{Su}, \mathrm{F})\end{array}$ \\
\hline $\begin{array}{l}\text { Kucharska- } \\
\text { Pietura } \\
\text { et al. [26] }\end{array}$ & $\begin{array}{l}30 \mathrm{RBD} \\
30 \mathrm{LBD} \\
50 \mathrm{NC}\end{array}$ & MMSE & no & static photographs & H, Sa, F, A, Su, D & $\begin{array}{l}\text { labeling; } \\
\text { discrimination; } \\
\text { recognition }\end{array}$ & $\begin{array}{l}\text { in all the tasks, } \\
\mathrm{RBD}<\mathrm{LBD}<\mathrm{NC}\end{array}$ \\
\hline $\begin{array}{l}\text { Zgaljardic } \\
\text { and Borod } \\
{[43]}\end{array}$ & $\begin{array}{l}9 \text { RBD } \\
7 \text { LBD } \\
7 \mathrm{NC}\end{array}$ & $\begin{array}{l}\text { MDRS; BPD; } \\
\text { BDAE; } \\
\text { WAIS-R }\end{array}$ & yes & $\begin{array}{l}\text { static photographs } \\
\text { (Pictures of Facial Affect) }\end{array}$ & $\begin{array}{l}\text { PE: H, I, Ple.Su } \\
\text { NE: Sa, F, A, D, } \\
\text { UnPle.Su }\end{array}$ & identification & $\mathrm{RBD}<\mathrm{LBD}<\mathrm{NC}$ \\
\hline $\begin{array}{l}\text { Karow et al. } \\
\text { [96] }\end{array}$ & $\begin{array}{l}10 \mathrm{RBD} \\
10 \mathrm{LBD} \\
5 \mathrm{NC}\end{array}$ & WAB & no & dynamic faces & $\mathrm{H}, \mathrm{Sa}, \mathrm{A}, \mathrm{N}$ & identification & $\mathrm{RBD}<\mathrm{LBD}<\mathrm{NC}$ \\
\hline $\begin{array}{l}\text { Rapcsak } \\
\text { et al. [36] }\end{array}$ & $\begin{array}{l}63 \mathrm{FBD} \\
80 \mathrm{NC}\end{array}$ & NA & no & static photographs & H, Sa, A, Su, F, D & matching & $\mathrm{FBD}<\mathrm{NC}(\mathrm{F})$ \\
\hline $\begin{array}{l}\text { Mandal } \\
\text { et al. [35] }\end{array}$ & $\begin{array}{l}30 \mathrm{RBD} \\
30 \mathrm{LBD} \\
50 \mathrm{NC}\end{array}$ & EHS & no & static photographs & $\mathrm{H}, \mathrm{Sa}, \mathrm{F}, \mathrm{A}$ & matching & $\mathrm{RBD}<\mathrm{LBD}<\mathrm{NC}$ \\
\hline Borod et al. [44] & $\begin{array}{l}11 \mathrm{RBD} \\
10 \mathrm{LBD} \\
15 \mathrm{NC}\end{array}$ & $\begin{array}{l}\text { MDRS; BDAE; } \\
\text { BNT; BPD; } \\
\text { BVFD }\end{array}$ & yes & $\begin{array}{l}\text { static photographs } \\
\text { (Pictures of Facial Affect) }\end{array}$ & $\begin{array}{l}\text { PE: H, I, Ple.Su } \\
\text { NE: Sa, F, A, D, } \\
\text { UnPle.Su }\end{array}$ & $\begin{array}{l}\text { discrimination; } \\
\text { identification } \\
\text { (Likert) }\end{array}$ & $\begin{array}{l}\text { identification: } \\
\text { RBD < LBD < NC } \\
\text { discrimination: } \\
\text { no group differences }\end{array}$ \\
\hline $\begin{array}{l}\text { Vuilleumier } \\
\text { et al. [97] }\end{array}$ & $1 \mathrm{RBD}$ & $\begin{array}{l}\text { MMSE; } \\
\text { WAIS-R }\end{array}$ & no & static photographs & H, Su, A, Sa, F, D & $\begin{array}{l}\text { identification; } \\
\text { discrimination }\end{array}$ & $\begin{array}{l}\text { in both tasks, RBD } \\
\text { showed impairment }\end{array}$ \\
\hline $\begin{array}{l}\text { Peper and } \\
\text { Irle [65] }\end{array}$ & $\begin{array}{l}27 \mathrm{RBD} \\
37 \mathrm{LBD} \\
15 \mathrm{NC}\end{array}$ & NA & no & $\begin{array}{l}\text { static photographs } \\
\text { (Pictures of Facial Affect) }\end{array}$ & $\begin{array}{l}\mathrm{H}, \mathrm{Su}, \mathrm{A}, \mathrm{An}, \mathrm{Gr} \\
\mathrm{D}\end{array}$ & $\begin{array}{l}\text { categorization; } \\
\text { selection; matching; } \\
\text { discrimination; } \\
\text { decoding }\end{array}$ & $\mathrm{RBD}<\mathrm{LBD}<\mathrm{NC}$ \\
\hline $\begin{array}{l}\text { Schmitt et al. } \\
\text { [41] }\end{array}$ & $\begin{array}{l}27 \mathrm{RBD} \\
25 \mathrm{LBD} \\
26 \mathrm{NC}\end{array}$ & NA & no & dynamic faces & $\mathrm{H}, \mathrm{F}, \mathrm{N}$ & identification & $\mathrm{RBD}<\mathrm{LBD}<\mathrm{NC}(\mathrm{F})$ \\
\hline $\begin{array}{l}\text { Adolphs } \\
\text { et al. [98] }\end{array}$ & $\begin{array}{l}37 \mathrm{RBD} \\
15 \mathrm{NC}\end{array}$ & WAIS-IQ & no & $\begin{array}{l}\text { static photographs } \\
\text { (Pictures of Facial Affect) }\end{array}$ & $\mathrm{H}, \mathrm{Su}, \mathrm{F}, \mathrm{A}, \mathrm{D}, \mathrm{Sa}$ & judgment & $\mathrm{RBD}<\mathrm{NC}(\mathrm{F}$ and $\mathrm{Sa})$ \\
\hline Reilly et al. [67] & $\begin{array}{l}5 \text { RBD } \\
5 \text { LBD } \\
\text { (6-24-month- } \\
\text { old infants) }\end{array}$ & NA & no & $\begin{array}{l}\text { toys; wrist restraint } \\
\text { paradigm }\end{array}$ & $\begin{array}{l}\text { Pos and Neg } \\
\text { responsiveness }\end{array}$ & $\begin{array}{l}\text { infant attention } \\
\text { (video-recorded) }\end{array}$ & RBD < LBD (Pos) \\
\hline $\begin{array}{l}\text { Starkstein } \\
\text { et al. [28] }\end{array}$ & $\begin{array}{l}29 \mathrm{RBD} \\
30 \mathrm{NC}\end{array}$ & $\begin{array}{l}\text { MMSE; JHFI; } \\
\text { STC; HAD }\end{array}$ & no & $\begin{array}{l}\text { static photographs } \\
\text { (Pictures of Facial Affect) }\end{array}$ & $\mathrm{H}, \mathrm{Sa}, \mathrm{A}$ & $\begin{array}{l}\text { recognition; } \\
\text { identification }\end{array}$ & in both tasks, $\mathrm{RBD}<\mathrm{NC}$ \\
\hline $\begin{array}{l}\text { Weddell } \\
\text { [29] }\end{array}$ & $\begin{array}{l}27 \mathrm{RBD} \\
24 \mathrm{LBD} \\
15 \mathrm{NC}\end{array}$ & $\begin{array}{l}\text { WAIS-R; VIQ; } \\
\text { PIQ; WMS }\end{array}$ & no & $\begin{array}{l}\text { static photographs } \\
\text { (Pictures of Facial Affect) }\end{array}$ & $\begin{array}{l}\mathrm{H}, \mathrm{Sa}, \mathrm{A}, \mathrm{F}, \mathrm{D} \\
\mathrm{Su}, \mathrm{N}\end{array}$ & $\begin{array}{l}\text { matching; response; } \\
\text { categorization; } \\
\text { recognition }\end{array}$ & $\begin{array}{l}\text { in all the tasks, } \\
\mathrm{RBD}<\mathrm{LBD}<\mathrm{NC}\end{array}$ \\
\hline
\end{tabular}


Table 1 (continued)

\begin{tabular}{|c|c|c|c|c|c|c|c|}
\hline Reference & $\mathrm{n}$ & $\begin{array}{l}\text { Clinical } \\
\text { measures }\end{array}$ & $\begin{array}{l}\text { Control } \\
\text { task }\end{array}$ & Emotional stimuli & Emotions & Dependent variables & Results \\
\hline Borod et al. [64] & $\begin{array}{l}19 \mathrm{RBD} \\
21 \mathrm{NC}\end{array}$ & $\begin{array}{l}\text { WAIS-R; DRS; } \\
\text { BPRS }\end{array}$ & yes & $\begin{array}{l}\text { static photographs } \\
\text { (Pictures of Facial Affect) }\end{array}$ & $\begin{array}{l}\mathrm{H}, \mathrm{Sa}, \mathrm{A}, \mathrm{F}, \mathrm{D} \\
\mathrm{Su}, \mathrm{N}\end{array}$ & $\begin{array}{l}\text { discrimination; } \\
\text { identification }\end{array}$ & in both tasks, $\mathrm{RBD}<\mathrm{NC}$ \\
\hline $\begin{array}{l}\text { Young et al. } \\
{[71]}\end{array}$ & $\begin{array}{l}15 \mathrm{RBD} \\
19 \mathrm{LBD} \\
32 \mathrm{NC}\end{array}$ & NA & no & $\begin{array}{l}\text { static photographs } \\
\text { (Pictures of Facial Affect) }\end{array}$ & H, Sa, A, F, D, Su & $\begin{array}{l}\text { recognition; matching; } \\
\text { analysis }\end{array}$ & $\begin{array}{l}\text { in all the tasks, } \\
\mathrm{LBD}<\mathrm{RBD}<\mathrm{NC}\end{array}$ \\
\hline $\begin{array}{l}\text { Blonder } \\
\text { et al. [7] }\end{array}$ & $\begin{array}{l}10 \mathrm{RBD} \\
10 \mathrm{LBD} \\
10 \mathrm{NC}\end{array}$ & NA & no & static photographs & $\mathrm{H}, \mathrm{Sa}, \mathrm{A}, \mathrm{F}, \mathrm{N}$ & $\begin{array}{l}\text { discrimination; } \\
\text { labeling; matching }\end{array}$ & $\begin{array}{l}\text { in all the tasks, } \\
\mathrm{RBD}<\mathrm{LBD}<\mathrm{NC}\end{array}$ \\
\hline $\begin{array}{l}\text { Mandal et al. } \\
{[63]}\end{array}$ & $\begin{array}{l}10 \mathrm{RBD} \\
10 \mathrm{LBD} \\
20 \mathrm{NC}\end{array}$ & EHS & no & static photographs & $\mathrm{H}, \mathrm{Sa}, \mathrm{A}, \mathrm{Su}, \mathrm{D}$ & matching; labeling & $\begin{array}{l}\text { in both tasks, } \\
\mathrm{RBD}<\mathrm{LBD}<\mathrm{NC} \\
(\mathrm{F} \text { and } \mathrm{A})\end{array}$ \\
\hline Borod et al. [91] & $\begin{array}{l}19 \mathrm{RBD} \\
21 \mathrm{NC}\end{array}$ & SADS-L & no & $\begin{array}{l}\text { static photographs } \\
\text { (Pictures of Facial Affect) }\end{array}$ & $\mathrm{H}, \mathrm{Sa}, \mathrm{A}, \mathrm{Su}, \mathrm{F}$ & $\begin{array}{l}\text { discrimination; } \\
\text { identification (Likert) }\end{array}$ & in both tasks, $\mathrm{RBD}<\mathrm{NC}$ \\
\hline $\begin{array}{l}\text { Cancelliere } \\
\text { and Kertesz } \\
{[45]}\end{array}$ & $\begin{array}{l}28 \mathrm{RBD} \\
18 \mathrm{LBD} \\
20 \mathrm{NC}\end{array}$ & NA & no & static photographs & $\mathrm{H}, \mathrm{Sa}, \mathrm{A}, \mathrm{N}$ & $\begin{array}{l}\text { identification; } \\
\text { elicitation; repetition; } \\
\text { identification }\end{array}$ & $\begin{array}{l}\text { in all the tasks, } \\
\mathrm{RBD}=\mathrm{LBD}=\mathrm{NC}\end{array}$ \\
\hline $\begin{array}{l}\text { Ehlers and } \\
\text { Dalby [99] }\end{array}$ & $\begin{array}{l}17 \mathrm{RBD} \\
5 \mathrm{LBD} \\
4 \mathrm{NC}\end{array}$ & $\begin{array}{l}\text { air } \\
\text { conduction } \\
\text { test }\end{array}$ & no & static photographs & J, A, Sor, F & response & $\mathrm{RBD}<\mathrm{LBD}<\mathrm{NC}$ \\
\hline Borod et al. [62] & $\begin{array}{l}12 \mathrm{RBD} \\
15 \mathrm{LBD} \\
16 \mathrm{NC}\end{array}$ & BDAE; PIQ & no & $\begin{array}{l}\text { static photographs } \\
\text { (Pictures of Facial Affect) }\end{array}$ & $\begin{array}{l}\text { PE: H, Ple.Su, } \\
\text { SA } \\
\text { NE: Sa, F, A, D, C }\end{array}$ & identification (Likert) & $\mathrm{RBD}<\mathrm{LBD}<\mathrm{NC}(\mathrm{NE})$ \\
\hline $\begin{array}{l}\text { Bowers } \\
\text { and Bauer } \\
{[61]}\end{array}$ & $\begin{array}{l}11 \mathrm{RBD} \\
11 \mathrm{LBD} \\
11 \mathrm{NC}\end{array}$ & NA & no & $\begin{array}{l}\text { static photographs } \\
\text { (Pictures of Facial Affect) }\end{array}$ & $\mathrm{H}, \mathrm{Sa}, \mathrm{A}, \mathrm{N}$ & $\begin{array}{l}\text { discrimination; } \\
\text { identification; } \\
\text { indication }\end{array}$ & $\begin{array}{l}\text { in all the tasks, } \\
\mathrm{RBD}<\mathrm{LBD}<\mathrm{NC}\end{array}$ \\
\hline $\begin{array}{l}\text { Kulikov and } \\
\text { Sidorova } \\
{[100]}\end{array}$ & $\begin{array}{l}17 \mathrm{RBD} \\
37 \mathrm{LBD} \\
31 \mathrm{NC}\end{array}$ & MMSE & no & $\begin{array}{l}\text { static photographs } \\
\text { (Pictures of Facial Affect) }\end{array}$ & H, Su, Sa, F, A, D & identification & $\mathrm{RBD}<\mathrm{LBD}<\mathrm{NC}$ \\
\hline $\begin{array}{l}\text { Dekosky } \\
\text { et al. [59] }\end{array}$ & $\begin{array}{l}9 \mathrm{RBD} \\
9 \mathrm{LBD} \\
9 \mathrm{NC}\end{array}$ & NA & no & static photographs & $\begin{array}{l}\mathrm{H}, \mathrm{Sa}, \mathrm{A}, \\
\text { indifferent }\end{array}$ & $\begin{array}{l}\text { discrimination; } \\
\text { identification }\end{array}$ & $\begin{array}{l}\text { in both the tasks, } \\
\mathrm{RBD}<\mathrm{LBD}<\mathrm{NC}\end{array}$ \\
\hline $\begin{array}{l}\text { Benowitz } \\
\text { et al. [42] }\end{array}$ & $\begin{array}{l}5 \mathrm{RBD} \\
4 \mathrm{LBD} \\
4 \mathrm{SB} \\
1 \mathrm{RH}\end{array}$ & WAIS-IQ & no & dynamic faces & $\begin{array}{l}\text { Pos, Neg, Dom, } \\
\text { Sub }\end{array}$ & identification & $\mathrm{RBD}<\mathrm{LBD}$ \\
\hline $\begin{array}{l}\text { Prigatano and } \\
\text { Pribram [60] }\end{array}$ & $\begin{array}{l}8 \mathrm{RBD} \\
7 \mathrm{LBD} \\
5 \text { bilateral } \\
10 \mathrm{NC}\end{array}$ & NA & no & $\begin{array}{l}\text { static photographs } \\
\text { (Pictures of Facial Affect) }\end{array}$ & $\mathrm{H}, \mathrm{Sa}, \mathrm{F}, \mathrm{A}$ & identification; recall & $\begin{array}{l}\mathrm{RBD}=\mathrm{LBD}=\text { bilateral }= \\
\mathrm{NC}\end{array}$ \\
\hline $\begin{array}{l}\text { Kolb and } \\
\text { Taylor [70] }\end{array}$ & $\begin{array}{l}35 \mathrm{RBD} \\
24 \mathrm{LBD} \\
20 \mathrm{NC}\end{array}$ & NA & no & $\begin{array}{l}\text { static photographs } \\
\text { (Pictures of Facial Affect) }\end{array}$ & $\begin{array}{l}\text { H, Sa, F, A, Su, } \\
\text { D, I }\end{array}$ & matching & $\mathrm{RBD}<\mathrm{LBD}<\mathrm{NC}$ \\
\hline
\end{tabular}

$\mathrm{A}=$ Anger; $\mathrm{An}=$ anxiety; $\mathrm{ASRH}=$ anterior strokes of right hemisphere; $\mathrm{BDAE}=$ Boston Diagnostic Aphasia Examination; $\mathrm{BDI}=\mathrm{Beck}$ Depression Inventory; BDST = Backward Digit Span Test; BG = patients with damage to the basal ganglia; BNT = Boston Naming Test; BPD = Benton Phoneme Discrimination Test; BPRS = Brief Psychiatric Rating Scale; BT = Ballons Test; BVFD = Benton Visual Form Discrimination Test; $\mathrm{C}=$ confusion; Cont = contentment; D = disgust; Dom = dominant; DRS = Depression Rating Scale; EBI = Extended Barthel Index; EHS = Edinburgh Handedness Inventory Scale; F = fear; FBD = focal brain damage; FDST = Forward Digit Span Test; GDS = Geriatric Depression Scale; Gr = grief; H = happy; HAD = Hospital Anxiety and Depression Scale; HVOT = Hooper Visual Organization Test; I = interest; Inte = interrogative; J = joy; JACFEE = Japanese and Caucasian Facial Expressions of Emotion; JHFI = Johns Hopkins Functioning Inventory; Likert = Likert scale; LSS = left subcortical stroke; MDRS = Mattis Dementia Rating Scale; $\mathrm{N}=$ neutral; NA = not available; NC = normal controls; NE = negative emotions; Neg = negative; PANAS = Positive and Negative Affect Schedule; PE = positive emotions; PIQ = Performance Intelligence Quotient; Ple.Su = pleasant surprise; Pos = positive; PSRH = posterior strokes of right hemisphere; RH = right hemispherectomy; RSS = right subcortical stroke; Sa = sad; $\mathrm{SA}=$ sexual arousal; SADS-L = Schedule for Affective Disorders and Schizophrenia - Lifetime Version; SB = split brain; Sor = sorrow; SS = patients with subcortical brain damage; SSS = Scandinavian Stroke Test; ST = Stroop Test; STC = Social Ties Checklist; Su = surprise; Sub = submissive; $\mathrm{T}=$ patients with damage to the thalamus; Unple.Su = unpleasant surprise; VFT = Verbal Fluency Test; VIQ = Verbal Intelligence Quotient; WAB = Western Aphasia Battery; WAIS-IQ = Wechsler Adult Intelligence Scale-Intelligence Quotient; WAIS-R = Wechsler Adult Intelligence Scale-Revised; WMS = Wechsler Memory Scale; ZVT-G = Number Relation Test. 
Table 2. Summary of emotion processing studies from the prosodic channel

\begin{tabular}{|c|c|c|c|c|c|c|c|}
\hline Reference & $\mathrm{n}$ & $\begin{array}{l}\text { Clinical } \\
\text { measures }\end{array}$ & $\begin{array}{l}\text { Control } \\
\text { task }\end{array}$ & Emotional stimuli & Emotions & Dependent variables & Results \\
\hline $\begin{array}{l}\text { Blonder } \\
\text { et al. [33] }\end{array}$ & $\begin{array}{l}12 \mathrm{RBD} \\
9 \mathrm{NC}\end{array}$ & GDS; PANAS & no & sentence pairs & $\mathrm{H}, \mathrm{Sa}, \mathrm{A}, \mathrm{F}, \mathrm{N}$ & $\begin{array}{l}\text { discrimination; } \\
\text { matching }\end{array}$ & $\begin{array}{l}\text { in both tasks, } \\
\text { RBD }<\text { NC }\end{array}$ \\
\hline $\begin{array}{l}\text { Ross and } \\
\text { Monnot [51] }\end{array}$ & $\begin{array}{l}16 \mathrm{RBD} \\
10 \mathrm{LBD} \\
43 \mathrm{NC}\end{array}$ & EHS & no & sentence pairs & H, Sa, A, Su, N, Di & $\begin{array}{l}\text { identification; } \\
\text { discrimination }\end{array}$ & $\begin{array}{l}\text { in all the tasks, } \\
\mathrm{RBD}<\mathrm{LBD}<\mathrm{NC}\end{array}$ \\
\hline $\begin{array}{l}\text { Rymarczyk and } \\
\text { Grabowska [52] }\end{array}$ & $\begin{array}{l}37 \mathrm{RBD} \\
26 \mathrm{NC}\end{array}$ & $\begin{array}{l}\text { HAD; } \\
\text { WAIS-R }\end{array}$ & no & sentence pairs & $\mathrm{H}, \mathrm{Sa}, \mathrm{A}$ & $\begin{array}{l}\text { discrimination; } \\
\text { identification }\end{array}$ & $\mathrm{RBD}<\mathrm{NC}$ \\
\hline $\begin{array}{l}\text { Harciarek } \\
\text { et al. [54] }\end{array}$ & $\begin{array}{l}30 \mathrm{RBD} \\
31 \mathrm{NC}\end{array}$ & MMSE & no & pseudo-sentences & $\mathrm{H}, \mathrm{Sa}, \mathrm{A}$ & identification & $\mathrm{RBD}<\mathrm{NC}$ \\
\hline $\begin{array}{l}\text { Nakhutina } \\
\text { et al. [27] }\end{array}$ & $\begin{array}{l}9 \mathrm{RBD} \\
8 \mathrm{LBD} \\
7 \mathrm{NC}\end{array}$ & $\begin{array}{l}\text { MDRS; SADS-L; } \\
\text { BDAE }\end{array}$ & yes & sentences & $\mathrm{H}, \mathrm{Sa}, \mathrm{A}, \mathrm{F}, \mathrm{N}$ & $\begin{array}{l}\text { judgment; accuracy; } \\
\text { intensity (Likert); } \\
\text { confidence (Likert) }\end{array}$ & $\begin{array}{l}\text { in all the tasks, } \\
\mathrm{RBD}=\mathrm{LBD}=\mathrm{NC}\end{array}$ \\
\hline Pell [30] & $\begin{array}{l}9 \mathrm{RBD} \\
11 \mathrm{LBD} \\
12 \mathrm{NC}\end{array}$ & MMSE & yes & pseudo-sentences & H, Ple.Su, A, D, Sa & $\begin{array}{l}\text { discrimination; } \\
\text { identification; } \\
\text { rating }\end{array}$ & $\begin{array}{l}\text { in all the tasks, } \\
\mathrm{RBD}<\mathrm{LBD}<\mathrm{NC}\end{array}$ \\
\hline $\begin{array}{l}\text { Yip et al. } \\
{[34]}\end{array}$ & $\begin{array}{l}14 \mathrm{SS} \\
14 \mathrm{NC}\end{array}$ & $\begin{array}{l}\text { FDST; BT; } \\
\text { BDST; ST; VFT; } \\
\text { HVOT }\end{array}$ & no & sentences & H, Sa, A, Su, D, F & $\begin{array}{l}\text { identification; } \\
\text { discrimination }\end{array}$ & $\begin{array}{l}\text { in both the tasks, } \\
\mathrm{SS}<\mathrm{NC}(\mathrm{F}, \mathrm{Sa}, \mathrm{D})\end{array}$ \\
\hline $\begin{array}{l}\text { Charbonneau } \\
\text { et al. [25] }\end{array}$ & $\begin{array}{l}15 \mathrm{RBD} \\
17 \mathrm{LBD} \\
16 \mathrm{NC}\end{array}$ & MMSE & no & sentence pairs & H, Sa, A, Su, D, F & discrimination & $\begin{array}{l}\mathrm{RBD}<\mathrm{LBD}<\mathrm{NC} \\
(\mathrm{F}, \mathrm{Sa}, \mathrm{A})\end{array}$ \\
\hline $\begin{array}{l}\text { Kucharska-Pietura } \\
\text { et al. [26] }\end{array}$ & $\begin{array}{l}30 \mathrm{RBD} \\
30 \mathrm{LBD} \\
50 \mathrm{NC}\end{array}$ & MMSE & no & sentences & H, Sa, F, A, Su, D & identification & $\mathrm{RBD}<\mathrm{LBD}<\mathrm{NC}$ \\
\hline $\begin{array}{l}\text { Walker } \\
\text { et al. [53] }\end{array}$ & $\begin{array}{l}8 \mathrm{RBD} \\
8 \mathrm{LBD} \\
8 \mathrm{NC}\end{array}$ & $\begin{array}{l}\text { WAB; } \\
\text { RICE }\end{array}$ & no & $\begin{array}{l}\text { congruent or } \\
\text { incongruent sentences }\end{array}$ & $\mathrm{H}, \mathrm{Sa}$ & identification & $\mathrm{RBD}<\mathrm{LBD}<\mathrm{NC}$ \\
\hline $\begin{array}{l}\text { Zgaljardic } \\
\text { and Borod } \\
{[43]}\end{array}$ & $\begin{array}{l}9 \mathrm{RBD} \\
7 \mathrm{LBD} \\
7 \mathrm{NC}\end{array}$ & $\begin{array}{l}\text { MDRS; BPD; } \\
\text { BDAE; WAIS-R }\end{array}$ & yes & sentences & $\begin{array}{l}\text { PE: H, I, Ple.Su } \\
\text { NE: Sa, F, A, D, UnPle. } \\
\text { Su }\end{array}$ & identification & $\mathrm{RBD}<\mathrm{LBD}<\mathrm{NC}$ \\
\hline $\begin{array}{l}\text { Karow et al. } \\
{[96]}\end{array}$ & $\begin{array}{c}10 \mathrm{RBD} \\
10 \mathrm{LBD} \\
5 \mathrm{NC}\end{array}$ & WAB & no & sentences & $\mathrm{H}, \mathrm{Sa}, \mathrm{A}, \mathrm{N}$ & identification & $\mathrm{RBD}<\mathrm{LBD}<\mathrm{NC}$ \\
\hline $\begin{array}{l}\text { Borod et al. } \\
{[44]}\end{array}$ & $\begin{array}{l}11 \mathrm{RBD} \\
10 \mathrm{LBD} \\
15 \mathrm{NC}\end{array}$ & $\begin{array}{l}\text { MDRS; BDAE; } \\
\text { BNT; BVFD; } \\
\text { BPD }\end{array}$ & yes & sentences & $\begin{array}{l}\text { PE: H, I, Ple.Su } \\
\text { NE: Sa, F, A, D, UnPle. } \\
\text { Su }\end{array}$ & $\begin{array}{l}\text { discrimination; } \\
\text { identification (Likert) }\end{array}$ & $\begin{array}{l}\text { identification: } \\
\text { RBD < LBD < NC } \\
\text { discrimination: no } \\
\text { group differences }\end{array}$ \\
\hline Pell [68] & $\begin{array}{l}9 \mathrm{RBD} \\
11 \mathrm{LBD} \\
10 \mathrm{NC}\end{array}$ & $\begin{array}{l}\text { air conduction } \\
\text { test }\end{array}$ & no & sentences & $\mathrm{H}, \mathrm{A}, \mathrm{Sa}, \mathrm{N}$ & identification & $\begin{array}{l}\text { in all the tasks, } \\
\mathrm{RBD}<\mathrm{LBD}<\mathrm{NC}\end{array}$ \\
\hline $\begin{array}{l}\text { Wertz et al. } \\
\text { [47] }\end{array}$ & $\begin{array}{l}20 \mathrm{RBD} \\
18 \mathrm{NC}\end{array}$ & NA & no & $\begin{array}{l}\text { sentences (read out by } \\
\text { the experimenter) }\end{array}$ & H, Sa, A, Su, T, Di & identification & $\mathrm{RBD}<\mathrm{NC}$ \\
\hline $\begin{array}{l}\text { Pell and Baum } \\
\text { [49] }\end{array}$ & $\begin{array}{l}9 \mathrm{RBD} \\
10 \mathrm{LBD} \\
10 \mathrm{NC}\end{array}$ & $\begin{array}{l}\text { air conduction } \\
\text { test }\end{array}$ & no & $\begin{array}{l}\text { filtered sentences; } \\
\text { pseudo-sentences }\end{array}$ & $\begin{array}{l}\text { intonation patterns: } \\
\text { AC: A, Sa, H } \\
\text { LC: Dec, Inte, Impe }\end{array}$ & $\begin{array}{l}\text { discrimination; } \\
\text { identification }\end{array}$ & $\mathrm{RBD}=\mathrm{LBD}=\mathrm{NC}$ \\
\hline $\begin{array}{l}\text { Ross et al. } \\
{[50]}\end{array}$ & $\begin{array}{l}12 \mathrm{RBD} \\
10 \mathrm{LBD} \\
16 \mathrm{NC}\end{array}$ & WAIS-R; WAB & no & filtered sentence pairs & H, Sa, A, Su, Di, N & discrimination & $\mathrm{RBD}<\mathrm{LBD}<\mathrm{NC}$ \\
\hline $\begin{array}{l}\text { Schmitt et al. } \\
\text { [41] }\end{array}$ & $\begin{array}{l}27 \mathrm{RBD} \\
25 \mathrm{LBD} \\
26 \mathrm{NC}\end{array}$ & NA & no & pseudo-sentences & $\mathrm{H}, \mathrm{F}, \mathrm{N}$ & identification & $\mathrm{RBD}<\mathrm{LBD}<\mathrm{NC}(\mathrm{F})$ \\
\hline $\begin{array}{l}\text { Starkstein } \\
\text { et al. [28] }\end{array}$ & $\begin{array}{l}29 \mathrm{RBD} \\
30 \mathrm{NC}\end{array}$ & $\begin{array}{l}\text { MMSE; JHFI; } \\
\text { STC; HAD }\end{array}$ & no & sentences & $\mathrm{H}, \mathrm{Sa}, \mathrm{A}$ & $\begin{array}{l}\text { recognition; } \\
\text { identification }\end{array}$ & $\begin{array}{l}\text { in both the tasks, } \\
\text { RBD }<\text { NC }\end{array}$ \\
\hline Weddell [29] & $\begin{array}{l}27 \mathrm{RBD} \\
24 \mathrm{LBD} \\
15 \mathrm{NC}\end{array}$ & $\begin{array}{l}\text { WAIS-R; VIQ; } \\
\text { PIQ; WMS }\end{array}$ & no & sentences & $\mathrm{H}, \mathrm{Sa}, \mathrm{A}, \mathrm{F}, \mathrm{D}, \mathrm{Su}, \mathrm{N}$ & $\begin{array}{l}\text { matching; response; } \\
\text { categorization; } \\
\text { recognition }\end{array}$ & $\begin{array}{l}\text { in all the tasks, } \\
\mathrm{RBD}<\mathrm{LBD}<\mathrm{NC}\end{array}$ \\
\hline
\end{tabular}


Table 2 (continued)

\begin{tabular}{|c|c|c|c|c|c|c|c|}
\hline Reference & $\mathrm{n}$ & $\begin{array}{l}\text { Clinical } \\
\text { measures }\end{array}$ & $\begin{array}{l}\text { Control } \\
\text { task }\end{array}$ & Emotional stimuli & Emotions & Dependent variables & Results \\
\hline $\begin{array}{l}\text { Lalande et al. } \\
\text { [55] }\end{array}$ & $\begin{array}{l}12 \mathrm{RBD} \\
10 \mathrm{LBD} \\
16 \mathrm{NC}\end{array}$ & NA & no & hummed utterances & J, Sa, F, Su, D, A & identification & $\mathrm{RBD}<\mathrm{LBD}<\mathrm{NC}$ \\
\hline $\begin{array}{l}\text { Van Lancker and } \\
\text { Sidtis [80] }\end{array}$ & $\begin{array}{l}13 \mathrm{RBD} \\
24 \mathrm{LBD} \\
37 \mathrm{NC}\end{array}$ & SSLE & no & sentences & $\mathrm{H}, \mathrm{Sa}, \mathrm{A}, \mathrm{Su}$ & matching & $\mathrm{RBD}=\mathrm{LBD}=\mathrm{NC}$ \\
\hline $\begin{array}{l}\text { Blonder et al. } \\
{[7]}\end{array}$ & $\begin{array}{l}10 \mathrm{RBD} \\
10 \mathrm{LBD} \\
10 \mathrm{NC}\end{array}$ & NA & no & sentence pairs & H, Sa, A, F, N & $\begin{array}{l}\text { discrimination; } \\
\text { identification }\end{array}$ & $\begin{array}{l}\text { in all the tasks, } \\
\mathrm{RBD}<\mathrm{LBD}<\mathrm{NC}\end{array}$ \\
\hline $\begin{array}{l}\text { Bradvik et al. } \\
{[72]}\end{array}$ & $\begin{array}{l}20 \mathrm{RBD} \\
18 \mathrm{NC}\end{array}$ & PTAT & no & sentences & A, H, Ind, Sa, Ast & identification & $\begin{array}{l}\text { in all the tasks, } \\
\text { RBD < NC }\end{array}$ \\
\hline $\begin{array}{l}\text { Twist et al. } \\
\text { [101] }\end{array}$ & $\begin{array}{l}12 \mathrm{RBD} \\
9 \mathrm{LBD} \\
10 \mathrm{NC}\end{array}$ & $\begin{array}{l}\text { air conduction } \\
\text { test }\end{array}$ & no & sentences & H, Sa, A, Su, D & discrimination & $\begin{array}{l}\mathrm{RBD}=\mathrm{LBD}=\mathrm{NC} \\
\text { (ERP component) }\end{array}$ \\
\hline $\begin{array}{l}\text { Borod et al. } \\
\text { [91] }\end{array}$ & $\begin{array}{l}19 \mathrm{RBD} \\
21 \mathrm{NC}\end{array}$ & SADS-L & no & sentences & H, Sa, A, Su, F & $\begin{array}{l}\text { discrimination; } \\
\text { identification (Likert) }\end{array}$ & $\begin{array}{l}\text { in both tasks, } \\
\mathrm{RBD}<\mathrm{NC}\end{array}$ \\
\hline $\begin{array}{l}\text { Cancelliere and } \\
\text { Kertesz [45] }\end{array}$ & $\begin{array}{l}28 \mathrm{RBD} \\
18 \mathrm{LBD} \\
20 \mathrm{NC}\end{array}$ & NA & no & sentences & $\mathrm{H}, \mathrm{Sa}, \mathrm{A}, \mathrm{N}$ & $\begin{array}{l}\text { identification; } \\
\text { elicitation; repetition; } \\
\text { identification }\end{array}$ & $\begin{array}{l}\text { in all the tasks, } \\
\mathrm{RBD}=\mathrm{LBD}=\mathrm{NC}\end{array}$ \\
\hline $\begin{array}{l}\text { Bowers et al. } \\
{[75]}\end{array}$ & $\begin{array}{l}9 \text { RBD } \\
8 \mathrm{LBD} \\
8 \mathrm{NC}\end{array}$ & NA & no & $\begin{array}{l}\text { neutral and filtered } \\
\text { sentences }\end{array}$ & $\begin{array}{l}\mathrm{H}, \mathrm{Sa}, \mathrm{A}, \\
\text { indifferent tone }\end{array}$ & categorization & $\mathrm{RBD}<\mathrm{LBD}<\mathrm{NC}$ \\
\hline $\begin{array}{l}\text { Ehlers and Dalby } \\
\text { [99] }\end{array}$ & $\begin{array}{l}17 \mathrm{RBD} \\
5 \mathrm{LBD} \\
4 \mathrm{NC}\end{array}$ & $\begin{array}{l}\text { air } \\
\text { conduction } \\
\text { test }\end{array}$ & no & sentences & J, A, Sor, F & identification & $\mathrm{RBD}<\mathrm{LBD}<\mathrm{NC}$ \\
\hline $\begin{array}{l}\text { Gorelick and } \\
\text { Ross [73] }\end{array}$ & $14 \mathrm{RBD}$ & NA & no & sentences; gestures & $\begin{array}{l}\mathrm{H}, \mathrm{A}, \mathrm{Sa}, \mathrm{Su} \\
\text { indifferent }\end{array}$ & matching; identification & $\begin{array}{l}\text { in both tasks, } \\
\text { impairment in RBD }\end{array}$ \\
\hline $\begin{array}{l}\text { House et al. } \\
{[79]}\end{array}$ & $\begin{array}{l}10 \mathrm{RBD} \\
10 \mathrm{LBD} \\
10 \mathrm{NC}\end{array}$ & HAD & no & sentences & Sa, N, Ex & judgment & $\mathrm{RBD}=\mathrm{LBD}=\mathrm{NC}$ \\
\hline $\begin{array}{l}\text { Kulikov and } \\
\text { Sidorova [100] }\end{array}$ & $\begin{array}{l}17 \mathrm{RBD} \\
37 \mathrm{LBD} \\
31 \mathrm{NC}\end{array}$ & MMSE & no & sentences & H, Su, Sa, F, A, D & identification & $\mathrm{RBD}<\mathrm{LBD}<\mathrm{NC}$ \\
\hline $\begin{array}{l}\text { Lebrun et al. } \\
{[81]}\end{array}$ & $1 \mathrm{RBD}$ & NA & no & sentences & $\mathrm{A}, \mathrm{Sa}, \mathrm{J}, \mathrm{Su}$ & identification & $\begin{array}{l}\text { no significant } \\
\text { impairment }\end{array}$ \\
\hline $\begin{array}{l}\text { Tompkins and } \\
\text { Flowers [46] }\end{array}$ & $\begin{array}{l}11 \mathrm{RBD} \\
11 \mathrm{LBD} \\
11 \mathrm{NC}\end{array}$ & NA & no & $\begin{array}{l}\text { filtered sentences; } \\
\text { filtered sentence pairs }\end{array}$ & $\mathrm{H}, \mathrm{A}, \mathrm{Af}$ & $\begin{array}{l}\text { discrimination; } \\
\text { identification }\end{array}$ & $\begin{array}{l}\text { in all the tasks, } \\
\mathrm{RBD}<\mathrm{LBD}<\mathrm{NC}\end{array}$ \\
\hline $\begin{array}{l}\text { Tompkins and } \\
\text { Mateer [102] }\end{array}$ & $\begin{array}{l}10 \mathrm{RBD} \\
8 \mathrm{LBD} \\
24 \mathrm{NC}\end{array}$ & WAIS-IQ & no & sentences & NA & judgment & $\mathrm{RBD}<\mathrm{LBD}<\mathrm{NC}$ \\
\hline $\begin{array}{l}\text { Denes et al. } \\
{[76]}\end{array}$ & $\begin{array}{c}15 \mathrm{RBD} \\
15 \mathrm{LBD} \\
6 \mathrm{NC}\end{array}$ & NA & no & vowels & H, F, A, Sa, D & $\begin{array}{l}\text { discrimination; } \\
\text { identification }\end{array}$ & $\begin{array}{l}\text { in both tasks, } \\
\mathrm{RBD}<\mathrm{LBD}<\mathrm{NC}\end{array}$ \\
\hline $\begin{array}{l}\text { Heilman et al. } \\
{[48]}\end{array}$ & $\begin{array}{l}8 \mathrm{RBD} \\
9 \mathrm{LBD} \\
15 \mathrm{NC}\end{array}$ & NA & no & filtered sentences & $\mathrm{H}, \mathrm{Sa}, \mathrm{A}$ & identification & $\mathrm{RBD}<\mathrm{LBD}<\mathrm{NC}$ \\
\hline $\begin{array}{l}\text { Hughes et al. } \\
{[103]}\end{array}$ & $\begin{array}{l}12 \mathrm{RBD} \\
7 \mathrm{NC}\end{array}$ & NA & no & $\begin{array}{l}\text { sentences; various } \\
\text { emotional gestures }\end{array}$ & $\begin{array}{l}\text { H, Sa, Su, A, Fla, H, A, } \\
\text { Su, Sa, N }\end{array}$ & $\begin{array}{l}\text { discrimination; } \\
\text { repetition; identification }\end{array}$ & $\begin{array}{l}\text { in all the tasks, } \\
\mathrm{RBD}<\mathrm{NC}\end{array}$ \\
\hline Ross [77] & 10 RBD & $\begin{array}{l}\text { air conduction } \\
\text { test }\end{array}$ & no & sentence & $\begin{array}{l}\text { H, Sa, A, Su, } \\
\text { indifferent, Di }\end{array}$ & identification & $\begin{array}{l}\text { RBD showed } \\
\text { impairment }\end{array}$ \\
\hline $\begin{array}{l}\text { Tucker et al. } \\
\text { [58] }\end{array}$ & $\begin{array}{l}11 \mathrm{RBD} \\
7 \mathrm{LBD} \\
8 \mathrm{NC}\end{array}$ & NA & no & sentences & $\begin{array}{l}\text { A, H, S, indifferent } \\
\text { tone }\end{array}$ & $\begin{array}{l}\text { recognition; } \\
\text { discrimination }\end{array}$ & $\begin{array}{l}\text { in all the tasks, } \\
\mathrm{RBD}<\mathrm{LBD}<\mathrm{NC}\end{array}$ \\
\hline $\begin{array}{l}\text { Schlanger et al. } \\
{[104]}\end{array}$ & $\begin{array}{l}20 \text { RBD } \\
40 \text { aphasic }\end{array}$ & NA & no & sentences & $\mathrm{H}, \mathrm{Sa}, \mathrm{A}$ & $\begin{array}{l}\text { identification; intensity } \\
\text { (Likert) }\end{array}$ & $\begin{array}{l}\text { RBD = aphasic } \\
\text { group }\end{array}$ \\
\hline $\begin{array}{l}\text { Heilman et al. } \\
{[74]}\end{array}$ & $\begin{array}{l}6 \mathrm{RBD} \\
6 \mathrm{LBD}\end{array}$ & NA & no & sentences & $\mathrm{H}, \mathrm{Sa}, \mathrm{A}$, indifferent & judgment & $\mathrm{RBD}<\mathrm{LBD}$ \\
\hline
\end{tabular}




\section{Stimulus Presentation and Response Options}

There were a number of dissimilarities in the presentation of a stimulus and in response options. Some of these differences include the length of time for which participants were exposed to stimuli, whether with [25-27, 34, 39] or without time restriction [32, 43, 54]. Kucharska-Pietura et al. [26] imposed a time restriction for presentation of approximately $10 \mathrm{~s}$, with an interval of $10 \mathrm{~s}$ between two consecutive photographs. All studies of facial, prosodic and lexical emotion recognition used an alternative forced choice response paradigm, but they varied in the choices given. Most gave the same number of response options as there were emotion categories. For example, if six different emotions were presented, there would be six response options.

\section{Control Tasks}

The inclusion of a control task for perceptual emotional processing tasks was not noted in most of the studies. Rather, discrimination tasks, in which participants indicate whether photographs of people are the same or different, or identification tasks, in which the participants are required to label the emotion associated with each face $[38,43,44]$, were used. In the studies of prosodic perception, participants were asked to listen to an audiotape which contained sentences $[30,43,44]$. Similarly, in lexical emotion recognition, tasks from the NYEB were used [58].

\section{Description of Research with Normal Controls and Brain Damage Patients}

The following studies were reviewed to assess the two emotion hypotheses regarding lateralization and valence (i.e., right hemisphere and valence) with respect to emotional perception, communication channel, hemisphere and valence. Studies involved individuals with brain damage and healthy normal controls. The results of these studies are summarized in tables 1-3.

\section{Facial Channel}

Patients with stroke often have a 'mask-like' face and rarely express facial emotions [3]. Thus, when stroke patients view a face expressing emotion, they may fail to mimic this facial expression since they cannot activate motor representations important for recognition $[3,8]$. The first formal studies of emotional impairments in the facial channel associated with stroke were initiated in the 1980s [42, 59-62]. In later years, several studies regarding the performance of patients with brain damage in perceiving facial expressions were reported. These studies showed that individuals with RBD experienced greater difficulty in processing emotional faces than individuals with LBD and normal controls [6, 7, 25, 26, 30, 35, 39, 41, 43, $44,48,54,63-70]$. In contrast, most studies found no differences between RBD and LBD patients in the processing of emotional faces $[27,31,45,60]$, and one study found that LBD

\section{(Footnote to table 2)}

$\mathrm{A}=$ Anger; $\mathrm{AC}=$ affective context; $\mathrm{Af}=$ afraid; Ast = astonishment; $\mathrm{BDAE}=$ Boston Diagnostic Aphasia Examination; $\mathrm{BDST}=\mathrm{Backward}$ Digit Span Test; BNT = Boston Naming Test; BPD = Benton Phoneme Discrimination Test; BT = Ballons Test; BVFD = Benton Visual Form Discrimination Test; $\mathrm{D}=$ disgust; $\mathrm{Dec}=$ declarative; $\mathrm{Di}=$ disinterest; $\mathrm{EHS}=$ Edinburgh Handedness Inventory Scale; $\mathrm{ERP}=$ event-related potential; Ex = excited; F = fear; FDST = Forward Digit Span Test; Fla = flatness; GDS = Geriatric Depression Scale; H = happy; HAD = Hospital Anxiety and Depression Scale; HVOT = Hooper Visual Organization Test; I = interest; Impe = imperative; Ind = indifference; Inte = interrogative; J = joy; JHFI = Johns Hopkins Functioning Inventory; LC = linguistic context; Likert = Likert scale; MDRS = Mattis Dementia Rating Scale; $\mathrm{N}=$ neutral; NA = not available; $\mathrm{NC}=$ normal controls; $\mathrm{NE}=$ negative emotions; PANAS = Positive and Negative Affect Schedule; $\mathrm{PE}=$ positive emotions; PIQ = Performance Intelligence Quotient; Ple.Su = pleasant surprise; PTAT = pure tone audiometry test; RICE = RIC Evaluation of Communication Problems in Right Hemisphere Dysfunctions; Sa = sad; SADS-L = Schedule for Affective Disorders and Schizophrenia - Lifetime Version; Sor = sorrow; SS = patients with subcortical brain damage; SSLE = Standard Speech-Language Evaluation; $\mathrm{ST}=$ Stroop Test; $\mathrm{STC}=$ Social Ties Checklist; $\mathrm{Su}=$ surprise; $\mathrm{T}=$ patients with damage to the thalamus; UnPle.Su = unpleasant surprise; VFT = Verbal Fluency Test; VIQ = Verbal Intelligence Quotient; WAB = Western Aphasia Battery; WAIS-IQ = Wechsler Adult Intelligence Scale-Intelligence Quotient; WAIS-R = Wechsler Adult Intelligence Scale-Revised; WMS = Wechsler Memory Scale. 
Table 3. Summary of emotion processing studies from the lexical channel

\begin{tabular}{|c|c|c|c|c|c|c|c|}
\hline Reference & $\mathrm{n}$ & $\begin{array}{l}\text { Clinical } \\
\text { measures }\end{array}$ & $\begin{array}{l}\text { Control } \\
\text { task }\end{array}$ & Emotional stimuli & Emotions & Dependent variables & Results \\
\hline $\begin{array}{l}\text { Zgaljardic } \\
\text { and Borod [43] }\end{array}$ & $\begin{array}{l}9 \mathrm{RBD} \\
7 \mathrm{LBD} \\
7 \mathrm{NC}\end{array}$ & $\begin{array}{l}\text { MDRS; } \\
\text { BPD; BDAE; } \\
\text { WAIS-R }\end{array}$ & yes & sentences, words & $\begin{array}{l}\text { PE: H, I, Ple.Su } \\
\text { NE: Sa, F, A, D, } \\
\text { UnPle.Su }\end{array}$ & identification & $\mathrm{RBD}<\mathrm{LBD}<\mathrm{NC}$ \\
\hline $\begin{array}{l}\text { Cicero et al. } \\
{[56]}\end{array}$ & $\begin{array}{l}11 \mathrm{RBD} \\
10 \mathrm{LBD} \\
15 \mathrm{NC}\end{array}$ & $\begin{array}{l}\text { MDRS; BDAE; } \\
\text { BPD }\end{array}$ & yes & sentences, words & $\begin{array}{l}\text { PE: H, I, Ple.Su } \\
\text { NE: Sa, F, A, D, } \\
\text { UnPle.Su }\end{array}$ & $\begin{array}{l}\text { identification; } \\
\text { discrimination }\end{array}$ & $\mathrm{RBD}<\mathrm{LBD}<\mathrm{NC}$ \\
\hline $\begin{array}{l}\text { Borod et al. } \\
{[44]}\end{array}$ & $\begin{array}{l}11 \mathrm{RBD} \\
10 \mathrm{LBD} \\
15 \mathrm{NC}\end{array}$ & $\begin{array}{l}\text { MDRS; BDAE; } \\
\text { BNT; BVFD; BPD }\end{array}$ & yes & sentences, words & $\begin{array}{l}\text { PE: H, I, Ple.Su } \\
\text { NE: Sa, F, A, D, } \\
\text { UnPle.Su }\end{array}$ & $\begin{array}{l}\text { discrimination; } \\
\text { identification } \\
\text { (Likert) }\end{array}$ & $\begin{array}{l}\text { identification: } \mathrm{RBD}<\mathrm{LBD}<\mathrm{NC} \\
\text { discrimination: no group } \\
\text { differences }\end{array}$ \\
\hline $\begin{array}{l}\text { Schmitt et al. } \\
\text { [41] }\end{array}$ & $\begin{array}{l}27 \mathrm{RBD} \\
25 \mathrm{LBD} \\
26 \mathrm{NC}\end{array}$ & NA & no & sentences & $\mathrm{H}, \mathrm{F}, \mathrm{N}$ & identification & $\mathrm{RBD}=\mathrm{LBD}=\mathrm{NC}$ \\
\hline Weddell [29] & $\begin{array}{l}27 \mathrm{RBD} \\
24 \mathrm{LBD} \\
15 \mathrm{NC}\end{array}$ & $\begin{array}{l}\text { WAIS-R; VIQ; } \\
\text { PIQ; WMS }\end{array}$ & no & sentences (written) & $\mathrm{H}, \mathrm{Sa}, \mathrm{A}, \mathrm{F}, \mathrm{D}, \mathrm{Su}, \mathrm{N}$ & $\begin{array}{l}\text { matching; response; } \\
\text { categorization; } \\
\text { recognition }\end{array}$ & $\mathrm{RBD}<\mathrm{LBD}<\mathrm{NC}$ \\
\hline Borod [6] & $\begin{array}{l}16 \mathrm{NC} \\
16 \mathrm{RBD} \\
16 \mathrm{LB}\end{array}$ & BDAE & yes & sentences, words & $\begin{array}{l}\text { PE: H, Ple.Su } \\
\text { NE: Sa, F, A, D, C }\end{array}$ & $\begin{array}{l}\text { identification; } \\
\text { discrimination }\end{array}$ & in both tasks, $\mathrm{RBD}<\mathrm{LBD}<\mathrm{NC}$ \\
\hline $\begin{array}{l}\text { Brownell } \\
\text { et al. [57] }\end{array}$ & $\begin{array}{l}16 \mathrm{RBD} \\
16 \mathrm{NC}\end{array}$ & NA & no & vignettes & $\mathrm{H}, \mathrm{A}$ & response & $\mathrm{RBD}<\mathrm{NC}$ \\
\hline $\begin{array}{l}\text { Lalande et al. } \\
\text { [55] }\end{array}$ & $\begin{array}{l}12 \mathrm{RBD} \\
10 \mathrm{LBD} \\
16 \mathrm{NC}\end{array}$ & NA & no & sentences & J, Sa, F, Su, D, A & $\begin{array}{l}\text { identification; } \\
\text { decision; indication }\end{array}$ & $\mathrm{RBD}=\mathrm{LBD}=\mathrm{NC}$ \\
\hline $\begin{array}{l}\text { Blonder et al. } \\
\text { [7] }\end{array}$ & $\begin{array}{l}10 \mathrm{RBD} \\
10 \mathrm{LBD} \\
10 \mathrm{NC}\end{array}$ & NA & no & sentences & $\mathrm{H}, \mathrm{Sa}, \mathrm{A}, \mathrm{F}, \mathrm{N}$ & $\begin{array}{l}\text { discrimination; } \\
\text { identification }\end{array}$ & $\begin{array}{l}\mathrm{RBD}<\mathrm{LBD}<\mathrm{NC}^{\mathrm{a}} \\
\text { no selective deficits }{ }^{\mathrm{b}}\end{array}$ \\
\hline $\begin{array}{l}\text { Ostrove et al. } \\
{[22]}\end{array}$ & $\begin{array}{l}12 \mathrm{RBD} \\
12 \mathrm{NC}\end{array}$ & NA & no & $\begin{array}{l}\text { sentences } \\
\text { (vignettes) }\end{array}$ & Pos, Neg, N & $\begin{array}{l}\text { categorization; } \\
\text { response }\end{array}$ & in both tasks, RBD < NC (NE) \\
\hline $\begin{array}{l}\text { Bihrle et al. } \\
\text { [105] }\end{array}$ & $\begin{array}{l}10 \mathrm{RBD} \\
17 \mathrm{LBD} \\
10 \mathrm{NC}\end{array}$ & BDAE & no & jokes, stories & NA & $\begin{array}{l}\text { identification } \\
\text { (Likert) }\end{array}$ & $\begin{array}{l}\text { in both conditions, } \\
\mathrm{RBD}<\mathrm{LBD}<\mathrm{NC}\end{array}$ \\
\hline $\begin{array}{l}\text { Semenza et al. } \\
{[84]}\end{array}$ & $\begin{array}{l}20 \mathrm{RBD} \\
23 \mathrm{LBD} \\
23 \mathrm{NC}\end{array}$ & NA & no & words & $\mathrm{H}, \mathrm{Sa}, \mathrm{D}, \mathrm{A}, \mathrm{F}$ & judgment & $\mathrm{RBD}<\mathrm{LBD}<\mathrm{NC}$ \\
\hline Etcoff [69] & $\begin{array}{l}12 \mathrm{RBD} \\
12 \mathrm{LBD} \\
12 \mathrm{NC}\end{array}$ & NA & no & words & $\begin{array}{l}\text { H, Su, Sa, F, A, D, N, } \\
\text { I, Cont }\end{array}$ & judgment & $\mathrm{RBD}=\mathrm{LBD}=\mathrm{NC}$ \\
\hline $\begin{array}{l}\text { Brownell } \\
\text { et al. [106] }\end{array}$ & $\begin{array}{l}12 \mathrm{RBD} \\
12 \mathrm{NC}\end{array}$ & NA & no & jokes & NA & identification & $\mathrm{RBD}<\mathrm{NC}$ \\
\hline $\begin{array}{l}\text { Kolb and } \\
\text { Taylor [70] }\end{array}$ & $\begin{array}{l}35 \mathrm{RBD} \\
24 \mathrm{LBD} \\
20 \mathrm{NC}\end{array}$ & NA & no & sentences & $\mathrm{H}, \mathrm{Sa}, \mathrm{F}, \mathrm{A}, \mathrm{Su}, \mathrm{D}, \mathrm{I}$ & matching & $\mathrm{LBD}<\mathrm{RBD}<\mathrm{NC}$ \\
\hline
\end{tabular}

$\mathrm{A}=$ Anger; $\mathrm{BDAE}=$ Boston Diagnostic Aphasia Examination; BNT = Boston Naming Test; $\mathrm{BPD}=$ Benton Phoneme Discrimination Test; BVFD = Benton Visual Form Discrimination Test; C = confusion; Cont = contentment; D = disgust; F = fear; H = happy; I = interest; J = joy; Likert = Likert scale; MDRS = Mattis Dementia Rating Scale; $\mathrm{N}=$ neutral; $\mathrm{NA}=$ not available; $\mathrm{NC}=$ normal controls; $\mathrm{NE}=$ negative emotions; Neg = negative; $\mathrm{PE}=$ positive emotions; $\mathrm{PIQ}=\mathrm{Performance}$ Intelligence Quotient; Ple.Su = pleasant surprise; Pos = positive; $\mathrm{Sa}=$ sad; $\mathrm{Su}$ = surprise; UnPle.Su = unpleasant surprise; VIQ = Verbal Intelligence Quotient; WAIS-R = Wechsler Adult Intelligence Scale-Revised; WMS = Wechsler Memory Scale.

${ }^{\mathrm{a}}$ Comprehension of sentences involving nonverbal expressions of emotion. ${ }^{\mathrm{b}}$ Comprehension of sentences involving emotional event.

patients were more impaired than RBD patients [71]. In the latter study, there were findings which provided support for the valence hypothesis. Braun et al. [39] and Yip et al. [34] showed evidence that RBD patients were more impaired, particularly in the recognition of negative emotional facial expressions. In studies by Borod et al. [44] and Mandal et al. [35], brain damage patients were typically required to identify and match a range of facial emotions expressed. As a result, RBD patients had impairments relative to patients with LBD for 
negative emotions, but there were no differences between the two groups for positive emotions. On the other hand, Charbonneau et al. [25] found that RBD patients were impaired in the recognition of positive emotions such as happiness and surprise. In a tachistoscopic study with normal controls, right hemisphere involvement was obtained for the perception of facial emotional expressions with photograph, cartoon and line drawing stimuli [37].

\section{Prosodic Channel}

For the prosodic channel, participants are usually required to discriminate whether two emotionally toned sentences are the same or different and to identify the affect being expressed. Clinical studies have demonstrated that deficits in the perception of emotional prosody are associated more frequently with the right than the left hemisphere $[25,26,30$, $41,44,51,58,72-74]$. Studies using an identification task found that RBD patients were significantly impaired relative to LBD patients and normal controls [44, 48, 75]. In studies using a discrimination task, RBD patients had impairment in the comprehension of emotional prosody task [58, 76, 77]. In a study by Ross and Monnot [78], different patterns of deficits were observed following left and right brain damage, suggesting that affective prosody is a dominant and lateralized function of the right hemisphere. In contrast, House et al. [79] found that RBD and LBD participants were equally impaired in emotional prosody when judging the emotion conveyed by a recorded voice on a seven-point Likert scale. This is consistent with findings reported by Heilman et al. [48], in which RBD and LBD patients were equally impaired on the nonemotional prosody task compared with normal controls. However, other studies showed no significant impairments among RBD and LBD patients [27, 49, 80, 81]. Some studies in which RBD patients were impaired at recognizing negative emotions from the prosodic task $[25,35,41]$ provided support for the valence hypothesis. In a dichotic listening study with normal controls, a left ear (i.e., right hemisphere) involvement was found for the identification of prosodic information conveyed by speech stimuli from the NYEB [82].

\section{Lexical Channel}

One of the earliest studies was conducted by Wechsler [83], in which RBD patients had more difficulty than LBD patients in recalling emotional stories. Semenza et al. [84] reported that RBD patients showed greater impairment in processing emotional words relative to normal controls and LBD patients on a perceptual task involving analysis of hierarchical clustering. Ostrove et al. [22] showed that individuals with RBD had difficulties in understanding vignettes describing various social and emotional sets among positive, negative and neutral situations. Blonder et al. [7] found that RBD patients were impaired relative to LBD patients in making judgments about the emotional content of sentences involving nonverbal expressions of emotions. In a series of experiments with normal controls, Borod et al. [44] found that the presentation of emotional words and sentences during identification and discrimination tasks resulted in impairment of the right hemisphere. A study by Zgaljardic and Borod [43] reported that RBD patients were impaired relative to LBD patients and normal controls on identification tasks involving emotional words and sentences, as well as on nonemotional control tasks from the NYEB. In one study, the LBD group showed greater impairment on a sentence matching test where the subjects was asked to describe the emotion of a person in each sentence [70].

In a review of the normal control literature, there were findings of right and left hemisphere mediation in lexical emotional perception, which also support the valence hypothesis [85]. In a recent review of the behavioral and neuroimaging literature, the findings provided partial support for the valence hypothesis [86]. Additional support for the valence hypothesis can also be found in a recent functional magnetic resonance imaging study with normal controls [87]. This study demonstrated significant right hemisphere activation for negative 


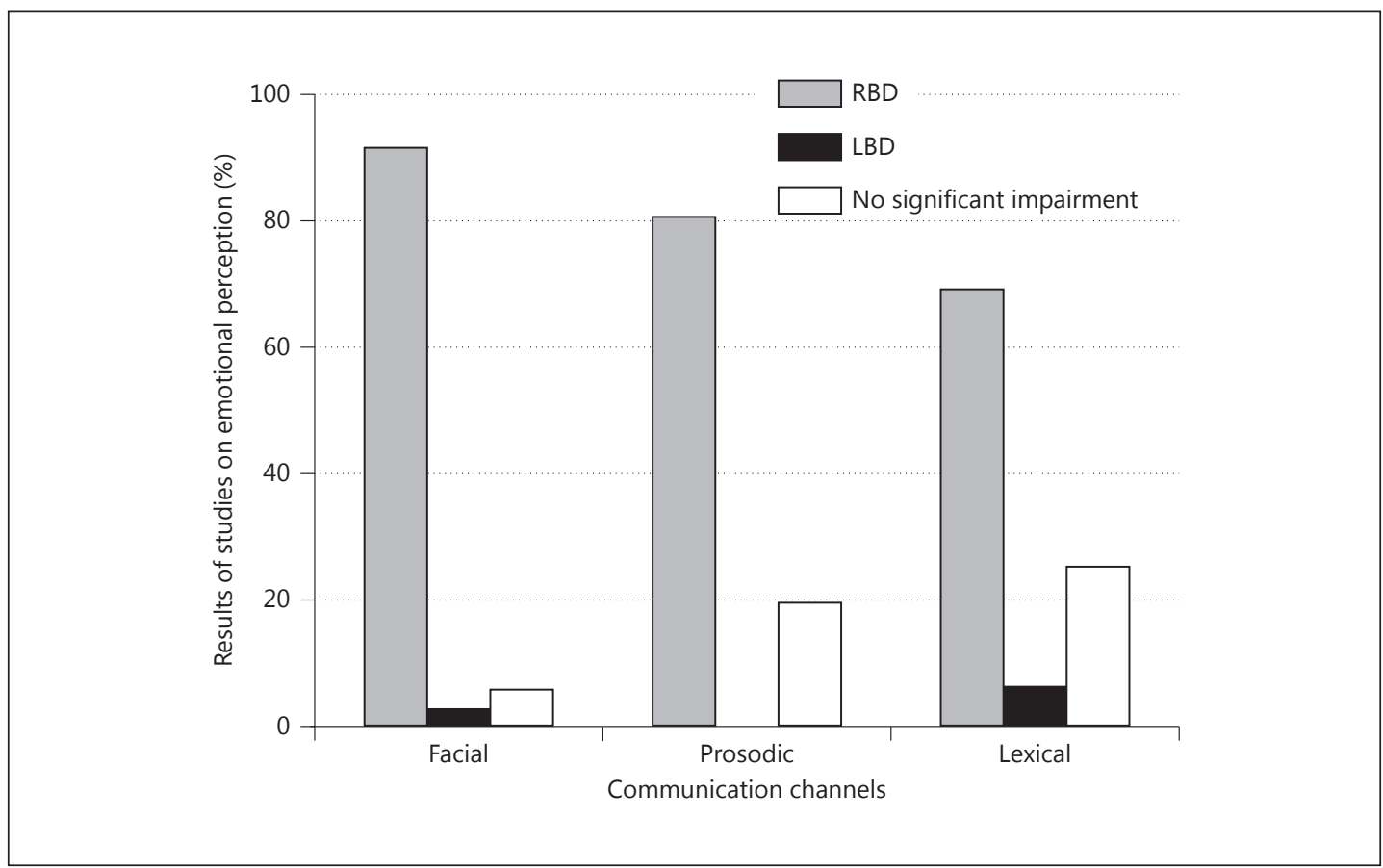

Fig. 2. Comparison of emotional processing deficits in brain-damaged patients.

emotion, but not for neutral, words. In a study by Graves et al. [88], healthy male subjects processed emotional words more accurately than nonemotional words in the right hemisphere, whereas neither men nor women showed differences between the two types of words in the left hemisphere. This finding suggests a special role for the right hemisphere in the perception of emotional words.

\section{Interchannel Relationships among Communication Channels}

There has been considerable work examining hemispheric specialization for emotional perception focused on three communication channels in parallel in both brain damage patients and normal controls $[6,70,89,90]$. In these studies, brain-damaged participants were required to identify emotion expressed in a facial stimulus item or to discriminate between two emotionally toned stimuli. The results from these studies were associated more frequently with pathology of the right than of the left hemisphere. The majority of these studies indicated that there was a significant correlation between the facial and prosodic channels, as compared to correlations with the lexical channel [7, 41, 43, 44, 54]. In a similar vein, studies looking at emotional behavior showed that the right hemisphere hypothesis of emotional processing is supported by examining the emotional perception for facial and prosodic channels $[27,68,91]$. However, studies involving normal participants typically examined facial, prosodic and lexical communication channels in parallel [92, 93]. Borod et al. [44] demonstrated that RBD patients were significantly impaired relative to LBD patients and normal controls across channels and valence, supporting the right hemisphere hypothesis.

\section{Reporting of Analysis and Outcomes}

A total of 92 studies were analyzed in this review. Among these studies, 35 discussed the facial channel, 41 studied the prosodic channel, and the remaining 16 reported on the lexical 
channel. In facial perception studies, 91\% (32/35) of the studies showed impairment in patients with RBD, 3\% (1/35) showed impairment in patients with LBD, and the remaining $6 \%(2 / 35)$ showed no significant impairment. For prosodic perception, 80\% (33/41) of the studies showed impairment in patients with RBD and 20\% (8/41) showed no significant impairment. For lexical perception, 69\% (11/16) of the studies showed impairment in patients with RBD, 6\% (1/16) showed impairment in patients with LBD, and the remaining $25 \%(4 / 16)$ showed no significant impairment. In summary, although impairment for emotion perception was more frequently observed in individuals with RBD than with LBD, there were a very fair number of studies that found no differences between the two patients groups (fig. 2).

\section{Discussion}

This review provides an analysis of the literature on emotional processing impairments in stroke patients. Our review supports the hypothesis that RBD patients have impairment relative to patients with LBD, normal control participants or both in processing facial and prosodic emotional stimuli. Certain results in the reviewed studies appear to be relatively robust and consistent with the behavioral literature involving facial and prosodic emotional perception in normal controls. For example, a recent review indicated that for facial and prosodic channels, the majority of observations revealed a greater involvement of right than left hemisphere specialization, regardless of emotional valence [86]. Taken together, these findings suggest that the right hemisphere is more involved in facial and prosodic emotional stimuli than the left hemisphere. In terms of lexical emotion perception, our review of studies involving individuals with brain damage supports right hemisphere specialization. These findings do not accord with the general literature. Specifically, in normal controls, evidence for the lexical channel demonstrated that there is relatively more involvement of the right than the left hemisphere, implicating some left hemisphere involvement in language processing [86]. The inconsistency between these findings for the two groups may result from difficulty in separating emotion from language processing in studies using normal control participants [85].

In addition, the method of stimulus presentation and task demands may impact emotion recognition results. Most of the earlier studies investigated emotion perception across all communication channels with the discrimination, identification, indication, judgment or imitation tasks using basic emotions such as happiness, sadness, anger, fear, disgust, surprise, neutral emotion or a combination. However, the studies did not obtain uniform results regarding the contribution of each cerebral hemisphere to emotion processing [34, 39, 63]. Other clinical studies provide results indicating equal impairment $[27,45,49,79,80]$. Many of the emotional processing clinical studies used a single modality of stimulus presentation, such as facial, prosodic or lexical, with few researchers attempting to explore the combination of the three. This variation between studies linked to different paradigms used for emotion recognition, even within a modality, led to divergent findings. Moreover, our understanding of emotion processing mechanisms remains too rudimentary to offer a principled account of this process.

In conclusion, our review of the literature on patients with brain damage and normal controls in which behavioral techniques were used point to right hemisphere dominance in the processing of emotion. Thus, the right hemisphere hypothesis was strongly supported; less evidence emerged for the valence hypothesis. The lateralization findings for emotional perception were stronger for the facial and prosodic channels than for the lexical channel. The conflicting findings across the three communication channels in the literature might be 
attributed to several different factors. The first set of factors concerns characteristics of brain damage patients themselves (disease measure stages, cognitive impairment, medication, disease duration, illness severity, behavior symptoms and different most affected side), whereas the second set of factors concerns aspects of the emotional task (stimulus modalities, task type and type of emotion). Without using a suitable emotional task, it is difficult to ascertain whether participants' poor performance with tasks of emotion recognition is due to an inability to identify emotions or whether their difficulties arise from decision making, categorization skills or the inability to identify the most salient features defining category boundaries. Indeed, the extent of emotion recognition in stroke patients appears to be correlated with a variety of interpersonal difficulties, such as complaints of frustration in social relations, feelings of social discomfort, desires to connect with others, feelings of social disconnection and use of controlling behaviors [94, 95]. Future research should aim to explore the need for large, longitudinal studies of emotion recognition in brain damage patients that are informed by emerging 'neurobiological data' together with a 'suitable methodological' approach, and which use consistent measures of disease stage and overall severity as well as uniform assessment instruments. In additional studies, it will be important to include more ecological types of emotion processing beyond the relatively artificial scenario of a forced choice recognition protocol.

\section{Acknowledgment}

The authors would like to thank the Ministry of Higher Education, Malaysia (grant number 9003-00341) for financially supporting this research work.

\section{References}

1 Truelsen T, Begg S, Mathers C: The global burden of cerebrovascular disease. 2000. http://www.who.int/ healthinfo/statistics/bod_cerebrovasculardiseasestroke.pdf (accessed January 1, 2012).

- 2 Wagle J, Farner L, Flekkoy K, Wyller TB, Sandvik L, Fure B, Stensrod B, Engedal K: Early post-stroke cognition in stroke rehabilitation patients predicts functional outcome at 13 months. Dement Geriatr Cogn Disord 2011; 31:379-387.

3 Borod J, Madigan NK: Neuropsychology of emotion and emotional disorders: an overview and research directions; in Borod J (ed): The Neuropsychology of Emotion. New York, Oxford University Press, 2000, pp 3-28.

$\checkmark$ Erhan H, Ochoa E, Borod J, Feinberg T: Consequences of right cerebrovascular accident on emotional functioning: diagnostic and treatment implications. CNS Spectr 2000;5:25-38.

5 Goodglass H, Kaplan E: The Assessment of Aphasia and Related Disorders. Philadelphia, Lea \& Febiger, 1983.

- 6 Borod J: Interhemispheric and intrahemispheric control of emotion: a focus on unilateral brain damage. J Consult Clin Psychol 1992;60:339-348.

7 Blonder LX, Bowers D, Heilman KM: The role of the right hemisphere in emotional communication. Brain 1991;114:1115-1127.

8 Borod J: The Neuropsychology of Emotion. New York, Oxford University Press, 2000.

- 9 Goldberg E, Costa LD: Hemisphere differences in the acquisition and use of descriptive systems. Brain Lang 1981;14:144-173.

10 Egelko S, Gordon WA, Hibbard MR, Diller L, Lieberman A, Holliday R, Ragnarsson K, Shaver MS, Orazem J: Relationship among CT scans, neurological exam, and neuropsychological test performance in right-braindamaged stroke patients. J Clin Exp Neuropsychol 1988;10:539-564.

$\checkmark 11$ Trotman SCA, Hammond GR: Lateral symmetry of the scalp distribution of somatosensory evoked potential amplitude. Brain Cogn 1989;10:132-147.

-12 Tucker DM, Roth DL, Bair TB: Functional connections among cortical regions: topography of EEG coherence. Electroencephalogr Clin Neurophysiol 1986;63:242-250.

13 Heilman KM: Discussant comments; in Borod JC, Buck R (eds): Asymmetries in Facial Expression: Method and Meaning. Symposium conducted at the International Neuropsychological Society, Pittsburgh, PA, 1982.

14 Tucker DM, Williamson P: Asymmetric neural control systems in human self-regulation. Psychol Rev 1984;91: 185-215. 
Yuvaraj et al.: Review of Emotion Recognition in Stroke Patients

15 Yokoyama K, Jennings R, Ackles P, Hood BS, Boiler F: Lack of heart rate changes during an attention-demanding task after right hemisphere lesions. Neurology 1987;37:624-630.

16 Heilman KM, Schwartz HD, Watson RT: Hypoarousal in patients with the neglect syndrome and emotional indifference. Neurology 1978;28:229-232.

17 Heller W: The neuropsychology of emotion: developmental patterns and implications for psychopathology; in Stein N, Leventhal BL, Trabasso T (eds): Psychological and Biological Approaches to Emotion. Hillsdale, Lawrence Erlbaum Associates, Inc, 1990, pp 167-211.

18 Silberman EK, Weingartner H: Hemispheric lateralization of function related to emotion. Brain Cogn 1986;5: 322-353.

19 Borod JC, Koff E, Buck R: The neuropsychology of facial expression in normal and brain-damaged subjects; in Blanck P, Buck R, Rosenthal R (eds): Nonverbal Communication in the Clinical Context. University Park, PA, Pennsylvania State University Press, 1986, pp 196-222.

20 Borod JC, Caron HS, Koff E: Asymmetry in positive and negative facial expressions: sex differences. Neuropsychologia 1981;19:819-824.

21 Ross ED, Homan RW, Buck R: Differential hemispheric lateralization of primary and social emotions: implications for developing a comprehensive neurology for emotions, repression, and the subconscious. Neuropsychiatry Neuropsychol Behav Neurol 1994;7:1-19.

-22 Ostrove J, Simpson TA, Gardner H: Beyond scripts: a note on the capacity of right hemisphere-damaged patients to process social and emotional content. Brain Cogn 1990;12:144-154.

23 Langer S, Pettigrew LC, Wilson JF, Blonder LX: Personality and social competency following unilateral stroke. J Int Neuropsychol Soc 1998;4:447-455.

24 Folstein MF, Folstein SE, McHugh PR: Mini-Mental State Examination: a practical method for grading the cognitive state of patients. Psychol Res 1975;12:189-198.

25 Charbonneau S, Scherzer BP, Aspirot D, Cohen H: Perception and production of facial and prosodic emotions by chronic CVA patients. Neuropsychologia 2003;41:605-613.

26 Kucharska-Pietura K, Phillips ML, Gernand W, David AS: Perception of emotions from faces and voices following unilateral brain damage. Neuropsychologia 2003;41:1082-1090.

27 Nakhutina L, Borod JC, Zgaljardic DJ: Posed prosodic emotional expression in unilateral stroke patients: recovery, lesion location, and emotional perception. Arch Clin Neuropsychol 2006;21:1-13.

28 Starkstein SE, Federoff JP, Price TR, Leiguarda RC, Robinson RG: Neuropsychological and neuroradiologic correlates of emotional prosody comprehension. Neurology 1994;44:515-522. Weddell RA: Effects of subcortical lesion site on human emotional behavior. Brain Cogn 1994;25:161-193. Pell MD: Cerebral mechanisms for understanding emotional prosody in speech. Brain Lang 2006;96:221-234. Cheung CCY, Lee TMC, Yip JTH, King KE, Li LSW: The differential effects of thalamus and basal ganglia on facial emotion recognition. Brain Cogn 2006;61:262-268.

-32 Harciarek M, Heilman KM: The contribution of anterior and posterior regions of the right hemisphere to the recognition of emotional faces. J Clin Exp Neuropsychol 2009;31:322-330.

-33 Blonder LX, Pettigrew LC, Kryscio RJ: Emotion recognition and marital satisfaction in stroke. J Clin Exp Neuropsychol 2012;34:634-642.

34 Yip JT, Leung KK, Li LS, Lee TM: The role of sub-cortical brain structures in emotion recognition. Brain Inj 2004;18:1209-1217.

-35 Mandal MK, Borod J, Asthana HS, Mohanty A, Mohanty S, Koff E: Effects of lesion variables and emotion type on the perception of facial emotion. J Nerv Ment Dis 1999;187:603-609.

-36 Rapcsak SZ, Galper SR, Comer JF, Reminger SL, Nielsen L, Kaszniak AW, Verfaellie M, Laguna JF, Labiner DM, Cohen RA: Fear recognition deficits after focal brain damage: a cautionary note. Neurology 2000;54:575-581. Strauss E, Moscovitch M: Perception of facial expression. Brain Lang 1981;13:308-332.

38 Borod J, Andelman F, Obler LK, Tweedy JR, Welkowitz J: Right hemisphere specialization for the identification of emotional words and sentences: evidence from stroke patients. Neuropsychologia 1992;30:827-844.

-39 Braun M, Traue HC, Frisch S, Deighton RM, Kessler H: Emotion recognition in stroke patients with left and right hemispheric lesion: results with a new instrument - the FEEL Test. Brain Cogn 2005;58:193-201.

40 Ekman P, Friesen WV: Pictures of Facial Affect. Palo Alto, Consulting Psychologists Press, 1976.

41 Schmitt JJ, Hartje W, Willmes K: Hemispheric asymmetry in the recognition of emotional attitude conveyed by facial expression, prosody and propositional speech. Cortex 1997;33:65-81.

42 Benowitz LI, Bear DM, Rosenthal R, Mesulam MM, Zaidel E, Sperry RW: Hemispheric specialization in nonverbal communication. Cortex 1983;19:5-11.

-43 Zgaljardic DJ, Borod J: Emotional perception in unilateral stroke patients: recovery, test stability, and interchannel relationships. Appl Neuropsychol 2002;9:159-172.

44 Borod J, Cicero BA, Obler LK, Welkowitz J, Erhan H, Santschi C, Grunwald C, Agosti RM, Whalen J: Right hemisphere emotional perception: evidence across multiple channels. Neuropsychology 1998;12:446-458.

45 Cancelliere AEB, Kertesz A: Lesion localization in acquired deficits of emotional expression and comprehension. Brain Cogn 1990;13:133-147.

46 Tompkins CA, Flowers CR: Perception of emotional intonation by brain-damaged adults: the influence of task processing levels. J Speech Hear Res 1985;28:527-538.

47 Wertz RT, Henschel CR, Auther LL, Ashford JR, Kirshner HS: Affective prosodic disturbance subsequent to right hemisphere stroke: a clinical application. J Neurolinguistics 1998;11:89-102. 
-48 Heilman KM, Bowers D, Speedie L, Coslett HB: Comprehension of affective and nonaffective prosody. Neurology 1984;34:917-920.

-49 Pell MD, Baum SR: Unilateral brain damage, prosodic comprehension deficits, and the acoustic cues to prosody. Brain Lang 1997;57:195-214.

50 Ross ED, Thompson RD, Yenkosky J: Lateralization of affective prosody in brain and the callosal integration of hemispheric language functions. Brain Lang 1997;56:27-54.

51 Ross ED, Monnot M: Affective prosody: what do comprehension errors tell us about hemispheric lateralization of emotions, sex and aging effects, and the role of cognitive appraisal. Neuropsychologia 2011;49:866-877.

52 Rymarczyk K, Grabowska A: Sex differences in brain control of prosody. Neuropsychologia 2007;45:921-930.

53 Walker JP, Daigle T, Buzzard M: Hemispheric specialization in processing prosodic structures: revisited. Aphasiology 2002;16:1155-1172.

54 Harciarek M, Heilman KM, Jodzio K: Defective comprehension of emotional faces and prosody as a result of right hemisphere stroke: modality versus emotion-type specificity. J Int Neuropsychol Soc 2006;12:774-781.

-55 Lalande S, Braun CMJ, Charlebois N, Whitaker HA: Effects of right and left hemisphere cerebrovascular lesions on discrimination of prosodic and semantic aspects of affect in sentences. Brain Lang 1992;42:165-186.

-56 Cicero B, Borod J, Santschi C, Obler L, Welkowitz J, Erhan H, Grunwald I, Agosti R: Emotional versus nonemotional lexical perception in patients with right and left brain damage. Neuropsychiatry Neuropsychol Behav Neurol 1999;12:255-264.

-57 Brownell HH, Carroll JJ, Rehak A, Wingfied A: The use of pronoun anaphora and speaker mood in the interpretation of conversational utterances by right hemisphere brain-damaged patients. Brain Lang 1992;43:121147.

58 Tucker DM, Watson RT, Heilman KM: Discrimination and evocation of affectively intoned speech in patients with right parietal disease. Neurology 1977;27:947-950.

59 Dekosky ST, Heilman KM, Bowers D, Valenstein E: Recognition and discrimination of emotional faces and pictures. Brain Lang 1980;9:206-214.

60 Prigatano GP, Pribram KH: Perception and memory of facial affect following brain injury. Percept Mot Skills 1982;54:859-869.

61 Bowers D, Bauer RM: Processing of faces by patients with unilateral hemisphere lesions. Brain Cogn 1985;4: 258-272.

62 Borod J, Koff E, Lorch MP, Nicholas M: The expression and perception of facial emotion in brain-damaged patients. Neuropsychologia 1986;24:169-180.

63 Mandal MK, Tandon SC, Asthana HS: Right brain damage impairs recognition of negative emotions. Cortex 1991;27:247-253.

64 Borod J, Martin CC, Alpert M, Brozgold A, Welkowitz J: Perception of facial emotion in schizophrenic and right brain-damaged patients. J Nerv Ment Dis 1993;181:494-502.

65 Peper M, Irle E: The decoding of emotional concepts in patients with focal cerebral lesions. Brain Cogn 1997; 34:360-387.

66 Adolphs R, Tranel D, Hamann S, Young AW, Calder AJ, Phelps EA, Anderson A, Lee GP, Damasio AR: Recognition of facial emotion in nine individuals with bilateral amygdala damage. Neuropsychologia 1999;37:1111-1117.

67 Reilly JS, Stiles J, Trauner JLD: Affective facial expression in infants with focal brain damage. Neuropsychologia 1995;33:83-99.

68 Pell MD: Recognition of prosody following unilateral brain lesion: influence of functional and structural attributes of prosodic contours. Neuropsychologia 1998;36:701-715.

-69 Etcoff NL: Perceptual and conceptual organization of facial emotions: hemispheric differences. Brain Cogn 1984;3:385-412.

70 Kolb B, Taylor L: Affective behavior in patients with localized cortical excisions: role of lesion site and side. Science 1981;214:89-91.

71 Young AW, Newcombe F, de Haan EH, Small M, Hay DC: Face perception after brain injury. Selective impairments affecting identity and expression. Brain 1993;116:941-959.

72 Bradvik B, Dravins C, Holtas S, Rosen I, Ryding E, Ingvar DH: Disturbances of speech prosody following right hemisphere infarcts. Acta Neurol Scand 1991;84:114-126.

73 Gorelick PB, Ross ED: The aprosodias: further functional-anatomical evidence for the organization of affective language in the right hemisphere. J Neurol Neurosurg Psychiatry 1987;50:553-560.

74 Heilman KM, Scholes R, Watson RT: Auditory affective agnosia. Disturbed comprehension of affective speech. J Neurol Neurosurg Psychiatry 1975;38:69-72.

75 Bowers D, Coslett HB, Bauer RM, Speedie LJ, Heilman KM: Comprehension of emotional prosody following unilateral hemispheric lesions: processing defect versus distraction defect. Neuropsychologia 1987;25:317328.

76 Denes G, Caldognetto EM, Semenza C, Vagges K, Zettin M: Discrimination and identification of emotions in human voice by brain-damaged subjects. Acta Neurol Scand 1984;69:154-162.

77 Ross ED: The aprosodias. Functional-anatomic organization of the affective components of language in the right hemisphere. Arch Neurol 1981;38:561-569.

78 Ross ED, Monnot M: Neurology of affective prosody and its functional-anatomic organization in right hemisphere. Brain Lang 2008;104:51-74. 
79 House A, Rowe D, Standen PJ: Affective prosody in the reading voice of stroke patients. J Neurol Neurosurg Psychiatry 1987;50:910-912.

80 Van Lancker D, Sidtis JJ: The identification of affective-prosodic stimuli by left- and right-hemisphere-damaged subjects: all errors are not created equal. J Speech Hear Res 1992;35:963-970.

81 Lebrun Y, Lessinnes A, Vresse LD, Leleux C: Dysprosody and the non-dominant hemisphere. Lang Sci 1985;7: 41-52.

82 Erhan H, Borod J, Tenke J, Bruder G: Identification of emotion in a dichotic listening task: event-related brain potential and behavioral findings. Brain Cogn 1998;37:286-307.

83 Wechsler A: The effect of organic brain disease on recall of emotionally charged versus neutral narrative texts. Neurology 1973;23:130-135.

84 Semenza C, Pasini M, Zettin M, Tonin P, Portolan P: Right hemisphere patients' judgements on emotions. Acta Neurol Scand 1986;74:43-50.

85 Borod J, Bloom R, Haywood DS: Verbal aspects of emotional communication; in Beeman M, Chiarello C (eds): Right Hemisphere Language Comprehension: Perspective from Cognitive Neuroscience. Mahwa, Lawrence Erlbaum Associates, Inc, 1998, pp 285-307.

86 Borod J, Zgaljardic DJ, Tabert M, Koff E: Asymmetries of emotional communication in normal subjects; in Boller F, Grafman J, Gainotti G (eds): Handbook of Neuropsychology: Emotional Behavior and Its Disorders. Oxford, Elsevier, 2001, pp 181-205.

87 Tabert M, Borod J, Tang C, Lange G, Wei T, Johnson R, Nusbaum A, Buchsbaum M: Differential amygdala activation during decision and recognition memory tasks using unpleasant words: a fMRI study. Neuropsychologia 2001;39:556-573.

88 Graves R, Landis T, Goodglass H: Laterality and sex differences for visual recognition of emotional and nonemotional words. Neuropsychologia 1981;19:95-102.

89 Heilman KM, Blonder LX, Bowers D, Valenstein E: Emotional disorders associated with neurological disease; in Heilman KM, Valenstein E (eds): Clinical Neuropsychology, ed 5. Oxford, Oxford University Press, 2011, pp 466-503.

90 Borod J: Emotional disorders/emotion; in Beaumont JG, Kenealy P, Rogers M (eds): The Blackwell Dictionary of Neuropsychology. Oxford, Blackwell, 1996, pp 312-320.

91 Borod J, Welkowitz J, Alpert M, Brozgold AZ, Martin C, Peselow E, Diller L: Parameters of emotional processing in neuropsychiatric disorders: conceptual issues and a battery of tests. J Commun Disord 1990;23:247-271.

92 Ekman P, Friesen W, O'Sullivan M, Scherer K: Relative importance of face, body, and speech in judgments of personality and affect. J Pers Soc Psychol 1980;38:270-277.

93 LoCastro J: Judgment of Emotional Communication in the Facial-Vocal-Verbal Channels. Unpublished doctoral dissertation, University of Maryland, College Park, 1972.

94 Clark US, Neargarder S, Cronin-Golomb A: Specific impairments in the recognition of emotional facial expressions in Parkinson's disease. Neuropsychologia 2008;46:2300-2309.

95 McCade D, Savage G, Naismith SL: Review of emotion recognition in mild cognitive impairment. Dement Geriatr Cogn Disord 2011;31:257-266.

96 Karow CM, Marquardt TP, Marshall RC: Affective processing in left and right hemisphere brain-damaged subjects with and without subcortical involvement. Aphasiology 2001;15:715-729.

97 Vuilleumier P, Ghika-Schmid F, Bogousslavsky J, Assal G, Regli F: Persistent recurrence of hypomania and prosopoaffective agnosia in a patient with right thalamic infarct. Neuropsychiatry Neuropsychol Behav Neurol 1998;11:40-44.

98 Adolphs R, Damasio H, Tranel D, Damasio AR: Cortical systems for the recognition of emotion in facial expressions. J Neurosci 1996;16:7678-7687.

99 Ehlers L, Dalby M: Appreciation of emotional expressions in the visual and auditory modality in normal and brain-damaged patients. Acta Neurol Scand 1987;76:251-256.

100 Kulikov MA, Sidorova OA: Identification of intonational and facial expressions of emotion by patients with organic lesions of the right or the left hemisphere. Sov Neurol Psychiatry 1985;18:75-87.

101 Twist DJ, Squires NK, Spielholz NI, Silverglide R: Event-related potentials in disorders of prosodic and semantic linguistic processing. Neuropsychiatry Neuropsychol Behav Neurol 1991;4:281-304.

-102 Tompkins CA, Mateer CA: Right hemisphere appreciation of prosodic and linguistic indications of implicit attitude. Brain Lang 1985;24:185-203.

103 Hughes CP, Chan JL, Su MS: Aprosodia in Chinese patients with right cerebral hemisphere lesions. Arch Neurol 1983;40:732-736.

104 Schlanger B, Schlanger P, Gerstman LJ: The perception of emotionally toned sentences by right hemisphere damaged and aphasic subjects. Brain Lang 1976;3:396-403.

105 Bihrle AM, Brownell HH, Powelson JA: Comprehension of humorous and nonhumorous materials by left and right brain-damaged patients. Brain Cogn 1986;5:399-341.

106 Brownell HH, Michel D, Powelson J, Gardner H: Surprise but not coherence: sensitivity to verbal humor in right-hemisphere patients. Brain Lang 1983;18:20-27. 